TRANSACTIONS OF THE

AMERICAN MATHEMATICAL SOCIETY

Volume 359, Number 4, April 2007, Pages 1685-1724

S 0002-9947(06)04052-9

Article electronically published on October 16, 2006

\title{
A SKEIN-LIKE MULTIPLICATION ALGORITHM FOR UNIPOTENT HECKE ALGEBRAS
}

\author{
NATHANIEL THIEM
}

\begin{abstract}
Let $G$ be a finite group of Lie type (e.g. $G L_{n}\left(\mathbb{F}_{q}\right)$ ) and $U$ a maximal unipotent subgroup of $G$. If $\psi$ is a linear character of $U$, then the unipotent Hecke algebra is $\mathcal{H}_{\psi}=\operatorname{End}_{\mathbb{C} G}\left(\operatorname{Ind}_{U}^{G}(\psi)\right)$. Unipotent Hecke algebras have a natural basis coming from double cosets of $U$ in $G$. This paper describes relations for reducing products of basis elements, and gives a detailed description of the implications in the case $G=G L_{n}\left(\mathbb{F}_{q}\right)$.
\end{abstract}

\section{INTRODUCTION}

Unipotent Hecke algebras interpolate between two classical Hecke algebras, the Gelfand-Graev Hecke algebra [St, Yo1] and the Yokonuma algebra Yo2 (a generalization of the Iwahori-Hecke algebra). These two classical algebras have not generally been studied from the same perspective, and an underlying philosophy of this paper is that techniques employed in the study of one classical algebra not only apply to the other, but also to all unipotent Hecke algebras.

The Gelfand-Graev Hecke algebra is a commutative algebra that has connections with Chevalley group representation theory [DM], unipotent orbits [Ka1], and Kloosterman sums [CS]. Despite being commutative, computing products in the standard double-coset basis is a challenging problem. The definition of a Hecke algebra implies $\left[\mathrm{CR}\right.$ that if $T_{h}$ and $T_{k}$ are two basis elements, then

$$
\text { (*) } T_{k} T_{h}=\sum_{v} c_{k h}^{v} T_{v} \text {, where } c_{k h}^{v}=\frac{1}{|U|^{2}} \sum_{\substack{u_{1}, u_{2}, u_{3}, u_{4} \in U \\ u_{1} k u_{2}=v u_{3} h^{-1} u_{4}}} \psi_{\mu}\left(u_{1}^{-1} u_{2}^{-1} u_{3} u_{4}\right) \text {, }
$$

but this formula is unhelpful for many applications. Using a geometric approach in $[\mathrm{Cu}$, Curtis analyzed which elements appear in the sums of (荬), but computing products in the Gelfand-Graev algebra still remains difficult.

This paper provides a uniform solution to the multiplication problem for Yokonuma Hecke algebras, Gelfand-Graev Hecke algebras, and all unipotent Hecke algebras. The idea is that in a unipotent Hecke algebra the $c_{h k}^{v}$ in (荬) are determined by generalizations of the braid-like relations of the Iwahori-Hecke algebra, and that the multiplication in any unipotent Hecke algebra can be done in a manner directly analogous to the way it is done in the Iwahori-Hecke algebra.

Received by the editors June 15, 2004 and, in revised form, January 21, 2005

2000 Mathematics Subject Classification. Primary 20C08; Secondary 05Exx.

Key words and phrases. Hecke algebra, Gelfand-Graev representation, unipotent Hecke algebra, Yokonuma Hecke algebra.

(C)2006 American Mathematical Society Reverts to public domain 28 years from publication 
Let $G$ be a finite Chevalley group with a maximal unipotent subgroup $U$. Suppose $\psi_{\mu}: U \rightarrow \mathbb{C}^{*}$ is a linear character of $U$. Then the unipotent Hecke algebra $\mathcal{H}\left(G, U, \psi_{\mu}\right)$ is

$$
\mathcal{H}_{\mu}=\operatorname{End}_{\mathbb{C} G}\left(\operatorname{Ind}_{U}^{G}\left(\psi_{\mu}\right)\right) \cong e_{\mu} \mathbb{C} G e_{\mu}, \quad \text { where } \quad e_{\mu}=\frac{1}{|U|} \sum_{u \in U} \psi_{\mu}\left(u^{-1}\right) u .
$$

Fix a subgroup $N \subseteq G$ of double coset representatives

$$
G=\bigsqcup_{v \in N} U v U, \quad \text { and let } \quad N_{\mu}=\left\{v \in N \mid e_{\mu} v e_{\mu} \neq 0\right\} .
$$

Then the set $\left\{e_{\mu} v e_{\mu} \mid v \in N_{\mu}\right\}$ is a basis for $\mathcal{H}_{\mu}$ [CR, Prop. 11.30].

\section{Examples.}

1. The Yokonuma Hecke algebra. If $\psi_{\mu}=\mathbb{1}$ is the trivial character, then $N_{\mathbb{1}}=$ $N$. Let $W=\left\langle s_{1}, s_{2}, \ldots, s_{\ell}\right\rangle$ be the Weyl group of $G$ and let $T=\left\langle h_{i}(t)\right| 1 \leq$ $\left.i \leq \ell, t \in \mathbb{F}_{q}^{*}\right\rangle$ be a maximal torus so that $N \cong T \rtimes W$. For $w \in W$ and $h \in T$, let $T_{h w}=e_{\mathbb{1}} h w e_{\mathbb{1}}$. By $\mathrm{Yo}_{0}$, the Yokonuma algebra $\mathcal{H}_{\mathbb{1}}$ has a basis $\left\{T_{v} \mid v \in N\right\}$ with relations

$$
\begin{aligned}
& T_{s_{i}} T_{w}= \begin{cases}T_{s_{i} w}, & \text { if } \ell\left(s_{i} w\right)=\ell(w)+1, \\
q^{-1} T_{h_{i}(-1) s_{i} w}+q^{-1} \sum_{t \in \mathbb{F}_{q}^{*}} T_{h_{i}(t) w}, & \text { if } \ell\left(s_{i} w\right)=\ell(w)-1, \\
& 1 \leq i \leq \ell, w \in W,\end{cases} \\
& T_{h} T_{w}=T_{h w}, \quad h \in T, w \in W, \\
& T_{h} T_{k}=T_{h k}, \quad h, k \in T,
\end{aligned}
$$

where if $w=s_{i_{1}} s_{i_{2}} \ldots s_{i_{r}} \in W$ for $r$ minimal, then $\ell(w)=r$. These relations give an "efficient" way to compute arbitrary products $\left(e_{\mathbb{1}} u e_{\mathbb{1}}\right)\left(e_{\mathbb{1}} v e_{\mathbb{1}}\right)$ in $\mathcal{H}_{\mathbb{1}}$.

2. The Gelfand-Graev Hecke algebra. If $\psi_{\mu}$ is in general position, then the Gelfand-Graev module $\operatorname{Ind}_{U}^{G}\left(\psi_{\mu}\right)$ is multiplicity free as a $G$-module (Yo1, St, Theorem 49]). The corresponding Hecke algebra $\mathcal{H}_{\mu}$ is therefore commutative. However, decomposing the product $\left(e_{\mu} u e_{\mu}\right)\left(e_{\mu} v e_{\mu}\right)$ into basis elements is more challenging than in the Yokonuma case $\mathrm{Ch}, \mathrm{Cu}, \mathrm{Ra}$.

Section 3 describes some of the subalgebra structure of unipotent Hecke algebras. The main results of the paper are in Section 4:

Theorem 4.1 and Corollary 3 give relations similar to those of the Yokonuma algebra (Example 1, above) for evaluating the product $\left(e_{\mu} u e_{\mu}\right)\left(e_{\mu} v e_{\mu}\right)$, with $u, v \in N_{\mu}$, in any unipotent Hecke algebra $\mathcal{H}_{\mu}$.

Section 5 applies the main results to the special case when $G=G L_{n}\left(\mathbb{F}_{q}\right)$, the general linear group over a finite field $\mathbb{F}_{q}$ with $q$ elements. Readers unfamiliar with the discourse of Chevalley groups may skip ahead to Section 5 (which is independent of Sections 3 and 4).

There are several natural ways to generalize unipotent Hecke algebras. In a series of papers Ka1, Ka2, Ka3 Kawanaka has analyzed a family of modules obtained by relaxing the maximality condition on $U$. There has also recently been a growing interest in a larger family of characters known as super characters An, ACDS. Seeing which aspects of the techniques associated with unipotent Hecke algebras extend to the Hecke algebras of these characters would be an interesting continuation of this work. 


\section{Preliminaries}

2.1. Finite Chevalley groups. Let $\mathfrak{g}=Z(\mathfrak{g}) \oplus \mathfrak{g}_{s}$ be a reductive Lie algebra, where $Z(\mathfrak{g})$ is the center of $\mathfrak{g}$ and $\mathfrak{g}_{s}=[\mathfrak{g}, \mathfrak{g}]$ is semisimple. If $\mathfrak{h}_{s}$ is a Cartan subalgebra of $\mathfrak{g}_{s}$, then $\mathfrak{h}=Z(\mathfrak{g}) \oplus \mathfrak{h}_{s}$ is a Cartan subalgebra of $\mathfrak{g}$. Let

$$
\mathfrak{h}^{*}=\operatorname{Hom}_{\mathbb{C}}(\mathfrak{h}, \mathbb{C}) \quad \text { and } \quad \mathfrak{h}_{s}^{*}=\operatorname{Hom}_{\mathbb{C}}\left(\mathfrak{h}_{s}, \mathbb{C}\right) .
$$

As an $\mathfrak{h}_{s}$-module, $\mathfrak{g}_{s}$ decomposes as

$$
\mathfrak{g}_{s} \cong \mathfrak{h}_{s} \oplus \bigoplus_{\alpha \in R}\left(\mathfrak{g}_{s}\right)_{\alpha}, \quad \text { where } \quad\left(\mathfrak{g}_{s}\right)_{\alpha}=\left\langle X \in \mathfrak{g}_{s} \mid[H, X]=\alpha(H) X, H \in \mathfrak{h}_{s}\right\rangle,
$$

and $R=\left\{\alpha \in \mathfrak{h}_{s}^{*} \mid \alpha \neq 0,\left(\mathfrak{g}_{s}\right)_{\alpha} \neq 0\right\}$ is the set of roots of $\mathfrak{g}_{s}$. Choose a set of simple roots $\left\{\alpha_{1}, \alpha_{2}, \ldots, \alpha_{\ell}\right\}$. This choice splits the set of roots $R$ into positive roots $R^{+}$and negative roots $R^{-}$with $R^{-}=-R^{+}$.

For each pair of roots $\alpha,-\alpha$, there exists a Lie algebra isomorphism $\phi_{\alpha}: \mathfrak{s l}_{2} \rightarrow$ $\left\langle\mathfrak{g}_{\alpha}, \mathfrak{g}_{-\alpha}\right\rangle$. Choose these isomorphisms such that if

$$
X_{\alpha}=\phi_{\alpha}\left(\begin{array}{ll}
0 & 1 \\
0 & 0
\end{array}\right) \in\left(\mathfrak{g}_{s}\right)_{\alpha}, \quad H_{\alpha}=\phi_{\alpha}\left(\begin{array}{cc}
1 & 0 \\
0 & -1
\end{array}\right) \in \mathfrak{h}_{s}, \quad X_{-\alpha}=\phi_{\alpha}\left(\begin{array}{ll}
0 & 0 \\
1 & 0
\end{array}\right) \in\left(\mathfrak{g}_{s}\right)_{-\alpha},
$$

then $\left\{X_{\alpha}, H_{\alpha_{i}} \mid \alpha \in R, 1 \leq i \leq \ell\right\}$ is a Chevalley basis of $\mathfrak{g}_{s}[\mathrm{Hu}$, Theorem 25.2].

Let $V$ be a finite-dimensional $\mathfrak{g}$-module such that $V$ has a $\mathbb{C}$-basis $\left\{v_{1}, v_{2}, \ldots, v_{r}\right\}$ that satisfies

(a) There exists a $\mathbb{C}$-basis $\left\{H_{1}, \ldots, H_{n}\right\}$ of $\mathfrak{h}$ such that

(1) $H_{\alpha_{i}} \in \mathbb{Z}_{\geq 0}$-span $\left\{H_{1}, \ldots, H_{n}\right\}$,

(2) $H_{i} v_{j} \in \mathbb{Z} v_{j}$ for all $i=1,2, \ldots, n$ and $j=1,2, \ldots, r$,

(3) $\operatorname{dim}_{\mathbb{Z}}\left(\mathbb{Z}-\operatorname{span}\left\{H_{1}, H_{2}, \ldots, H_{n}\right\}\right) \leq \operatorname{dim}_{\mathbb{C}}(\mathfrak{h})$.

(b) $\frac{X_{\alpha}^{n}}{n !} v_{i} \in \mathbb{Z}-\operatorname{span}\left\{v_{1}, v_{2}, \ldots, v_{r}\right\} \quad$ for $\alpha \in R, n \in \mathbb{Z}_{\geq 0}$ and $i=1,2, \ldots, r$.

(c) $\operatorname{dim}_{\mathbb{Z}}\left(\mathbb{Z}-\operatorname{span}\left\{v_{1}, v_{2}, \ldots, v_{r}\right\}\right) \leq \operatorname{dim}_{\mathbb{C}}(V)$.

(Condition (a) guarantees that $Z(\mathfrak{g})$ acts diagonally. If $Z(\mathfrak{g})=0$, then the existence of such a basis is guaranteed by a theorem of Kostant [Hu, Theorem 27.1].)

Let

$$
\mathfrak{h}_{\mathbb{Z}}=\mathbb{Z}-\operatorname{span}\left\{H_{1}, H_{2}, \ldots, H_{n}\right\} .
$$

The finite field $\mathbb{F}_{q}$ with $q$ elements has a multiplicative group $\mathbb{F}_{q}^{*}$ and an additive group $\mathbb{F}_{q}^{+}$. Let

$$
V_{q}=\mathbb{F}_{q}-\operatorname{span}\left\{v_{1}, v_{2}, \ldots, v_{r}\right\} .
$$

The finite reductive Chevalley group

$$
G_{V}=\left\langle x_{\alpha}(a), h_{H}(b) \mid \alpha \in R, H \in \mathfrak{h}_{\mathbb{Z}}, a \in \mathbb{F}_{q}, b \in \mathbb{F}_{q}^{*}\right\rangle
$$

is the subgroup of $G L\left(V_{q}\right)$ generated by the elements

$$
\begin{aligned}
& x_{\alpha}(a)=\sum_{n \geq 0} a^{n} \frac{X_{\alpha}^{n}}{n !}, \quad \text { and } \\
& h_{H}(b)=\operatorname{diag}\left(b^{\lambda_{1}(H)}, b^{\lambda_{2}(H)}, \ldots, b^{\lambda_{r}(H)}\right), \quad \text { where } H v_{i}=\lambda_{i}(H) v_{i} .
\end{aligned}
$$

Remark. If $\mathfrak{g}=\mathfrak{g}_{s}$, then $G_{V}=\left\langle x_{\alpha}(t) \mid \alpha \in R, t \in \mathbb{F}_{q}\right\rangle$.

Example. Suppose $\mathfrak{g}=\mathfrak{g l}_{2}$ and let

$$
V=\mathbb{C} \text {-span }\left\{\left(\begin{array}{l}
1 \\
0
\end{array}\right),\left(\begin{array}{l}
0 \\
1
\end{array}\right)\right\}
$$


be the natural $\mathfrak{g}$-module $\mathbb{C}^{2}$ given by matrix multiplication. Then $\mathfrak{h}$ has a basis

$$
\mathfrak{h}=\left\{\left(\begin{array}{ll}
a & 0 \\
0 & b
\end{array}\right) \mid a, b \in \mathbb{C}\right\}=\mathbb{C} \text {-span }\left\{\left(\begin{array}{ll}
1 & 0 \\
0 & 0
\end{array}\right),\left(\begin{array}{ll}
0 & 0 \\
0 & 1
\end{array}\right)\right\} .
$$

By direct computation,

$$
x_{\alpha}(t)=\left(\begin{array}{cc}
1 & t \\
0 & 1
\end{array}\right) \quad \text { and } \quad h_{\left(\begin{array}{cc}
a & 0 \\
0 & b
\end{array}\right)}(t)=\left(\begin{array}{cc}
t^{a} & 0 \\
0 & t^{b}
\end{array}\right) \quad \text { for } a, b \in \mathbb{Z},
$$

and $G_{V}=G L_{2}\left(\mathbb{F}_{q}\right)$ (the general linear group).

2.2. Important subgroups of a Chevalley group. Let $G=G_{V}$ be a Chevalley group defined with a $\mathfrak{g}$-module $V$ as above. The group $G$ contains a subgroup $U$ given by

$$
U=\left\langle x_{\alpha}(t) \mid \alpha \in R^{+}, t \in \mathbb{F}_{q}\right\rangle,
$$

which decomposes as

$$
U=\prod_{\alpha \in R^{+}} U_{\alpha}, \quad \text { where } \quad U_{\alpha}=\left\langle x_{\alpha}(t) \mid t \in \mathbb{F}_{q}\right\rangle,
$$

with uniqueness of expression for any fixed ordering of the positive roots St, Lemma 18]. For each $\alpha \in R^{+}$, the map

$$
\begin{aligned}
U_{\alpha} & \stackrel{\sim}{\longrightarrow} \mathbb{F}_{q}^{+} \\
x_{\alpha}(t) & \mapsto t
\end{aligned}
$$

is a group isomorphism.

For $\alpha, \beta \in R$, define the maps

$$
\begin{aligned}
& \begin{aligned}
s_{\alpha}: \quad \mathfrak{h}^{*} & \longrightarrow \mathfrak{h}^{*} \\
\gamma & \mapsto \gamma-\gamma\left(H_{\alpha}\right) \alpha \quad \text { and }
\end{aligned} \\
& s_{\alpha}: \mathfrak{h}=Z(\mathfrak{g}) \oplus \mathfrak{h}_{s} \longrightarrow \quad \mathfrak{h} \\
& H+H_{\beta} \quad \mapsto H+H_{\beta}-\beta\left(H_{\alpha}\right) H_{\alpha}{ }^{\circ}
\end{aligned}
$$

The Weyl group of $G$ is $W=\left\langle s_{\alpha} \mid \alpha \in R\right\rangle$ and has a presentation $W=\left\langle s_{1}, s_{2}, \ldots, s_{\ell} \mid s_{i}^{2}=1,\left(s_{i} s_{j}\right)^{m_{i j}}=1,1 \leq i \neq j \leq \ell\right\rangle, \quad m_{i j} \in \mathbb{Z}_{>0}, s_{i}=s_{\alpha_{i}}$. If $w=s_{i_{1}} s_{i_{2}} \cdots s_{i_{r}}$ with $r$ minimal, then the length of $w$ is $\ell(w)=r$.

Let $\mathfrak{h}_{\mathbb{Z}}$ be as in (2.1). If $q>3$, then the subgroup

$$
T=\left\langle h_{H}(t) \mid H \in \mathfrak{h}_{\mathbb{Z}}, t \in \mathbb{F}_{q}^{*}\right\rangle
$$

has its normalizer in $G$ given by

$$
N=\left\langle w_{\alpha}(t), h \mid \alpha \in R, h \in T, t \in \mathbb{F}_{q}^{*}\right\rangle, \quad \text { where } w_{\alpha}(t)=x_{\alpha}(t) x_{-\alpha}\left(-t^{-1}\right) x_{\alpha}(t) .
$$

If $\alpha \in R$, then $h_{H_{\alpha}}(t)=w_{\alpha}(t) w_{\alpha}(1)^{-1}$. Write $h_{\alpha}(t)=h_{H_{\alpha}}(t)$ and $h_{i}(t)=h_{\alpha_{i}}(t)$.

There is a natural surjection from $N$ onto the Weyl group $W$ with kernel $T$ given by

$$
\begin{aligned}
& \pi: \quad N \quad \longrightarrow \quad W \\
& w_{\alpha}(t) \mapsto s_{\alpha}, \quad \text { for } \alpha \in R, t \in \mathbb{F}_{q}^{*} \text {, } \\
& h \quad \mapsto \quad 1, \quad \text { for } h \in T \text {. }
\end{aligned}
$$

Suppose $v \in N$. Then for each minimal expression

$$
\pi(v)=s_{i_{1}} s_{i_{2}} \ldots s_{i_{r}}, \quad \text { with } \ell(\pi(v))=r,
$$


there is a unique $v_{T} \in T$ such that

$$
v=w_{i_{1}}(1) w_{i_{2}}(1) \cdots w_{i_{r}}(1) v_{T} .
$$

To simplify some notation in later sections, write

$$
v=v_{1} v_{2} \cdots v_{r} v_{T}, \quad \text { where } v_{i_{k}}=w_{i_{k}}(1) .
$$

2.3. Unipotent Hecke algebras. Let $G$ be a finite Chevalley group. Fix a nontrivial homomorphism $\psi: \mathbb{F}_{q}^{+} \rightarrow \mathbb{C}^{*}$. If

$$
\begin{aligned}
\mu: \quad R^{+} & \rightarrow \mathbb{F}_{q} \\
\alpha & \mapsto \mu_{\alpha}
\end{aligned} \quad \text { satisfies } \mu_{\alpha}=0 \text { for all } \alpha \text { not simple, }
$$

then the map

$$
\begin{array}{cccc}
\psi_{\mu}: & U & \longrightarrow & \mathbb{C}^{*} \\
& x_{\alpha}(t) & \mapsto & \left(\mu_{\alpha} t\right)
\end{array}
$$

is a linear character of $U$. With the exception of a few degenerate special cases of $G$ (which can be avoided if $q>3$ ), all linear characters of $U$ are of this form Yo1.5, Theorem 1].

The unipotent Hecke algebra $\mathcal{H}\left(G, U, \psi_{\mu}\right)$ is

$$
\mathcal{H}_{\mu}=\operatorname{End}_{\mathbb{C} G}\left(\operatorname{Ind}_{U}^{G}\left(\psi_{\mu}\right)\right)
$$

or viewed as a subset of $\mathbb{C} G$,

$$
\mathcal{H}_{\mu}=e_{\mu} \mathbb{C} G e_{\mu}, \quad \text { where } \quad e_{\mu}=\frac{1}{|U|} \sum_{u \in U} \psi_{\mu}\left(u^{-1}\right) u .
$$

Remark. Since $T$ is in the normalizer of $U$ in $G, T$ acts on the linear characters of $U$ by

$$
{ }^{h} \chi(u)=\chi\left(h u h^{-1}\right), \quad \text { where } u \in U, h \in T, \text { and } \chi: U \rightarrow \mathbb{C}^{*} .
$$

If two linear characters $\chi$ and $\gamma$ are in the same $T$-orbit, then $\mathcal{H}(G, U, \chi) \cong$ $\mathcal{H}(G, U, \gamma)$ (although the converse does not necessarily hold).

The group $G$ has a double-coset decomposition

$$
G=\bigsqcup_{v \in N} U v U \quad[\text { St, Theorem 4], }
$$

and if

$$
\begin{aligned}
N_{\mu} & =\left\{v \in N \quad \mid \quad e_{\mu} v e_{\mu} \neq 0\right\} \\
& =\left\{v \in N \mid u, v u v^{-1} \in U \text { implies } \psi_{\mu}(u)=\psi_{\mu}\left(v u v^{-1}\right)\right\},
\end{aligned}
$$

then the set $\left\{e_{\mu} v e_{\mu} \mid v \in N_{\mu}\right\}$ is a basis for $\mathcal{H}_{\mu}$ [CR, Prop. 11.30].

Examples (see also the Introduction).

1. The Yokonuma Hecke algebra. If $\mu_{\alpha}=0$ for all positive roots $\alpha$, then $\psi_{\mu}=$ $\mathbb{1}$ is the trivial character and $N_{\mathbb{1}}=N$. Let $T_{v}=e_{\mathbb{1}} v e_{\mathbb{1}}$ for $v \in N$, with $T_{i}=T_{w_{i}(1)}$ and $T_{H}(t)=T_{h_{H}(t)}$. If $v=v_{1} v_{2} \cdots v_{r} v_{T} \in N$ according to a minimal expression $s_{i_{1}} s_{i_{2}} \cdots s_{i_{r}} \in W($ as in (2.8) $)$, then

$$
T_{v}=T_{i_{1}} T_{i_{2}} \cdots T_{i_{r}} T_{v_{T}} .
$$


Thus, the Yokonuma algebra $\mathcal{H}_{\mathbb{1}}$ has generators $\left\{T_{i}, T_{h} \mid 1 \leq i \leq \ell, h \in T\right\}$ (see [Y02]) with relations

$$
\begin{aligned}
T_{i}^{2}=q^{-1} T_{H_{\alpha_{i}}}(-1) & +q^{-1} \sum_{t \in \mathbb{F}_{q}^{*}} T_{H_{\alpha_{i}}}\left(t^{-1}\right) T_{i}, & & 1 \leq i \leq \ell, \\
\underbrace{T_{i} T_{j} T_{i} \cdots}_{m_{i j} \text { terms }} & =\underbrace{T_{j} T_{i} T_{j} \cdots}_{m_{i j} \text { terms }}, & & \left(s_{i} s_{j}\right)^{m_{i j}}=1, \\
T_{i} T_{h} & =T_{s_{i} h} T_{i}, & & h \in T, \\
T_{h} T_{k} & =T_{h k}, & & h, k \in T .
\end{aligned}
$$

These relations give an "efficient" way to compute arbitrary products $T_{u} T_{v}$ in $\mathcal{H}_{\mathbb{1}}$. There is a surjective map from the Yokonuma algebra onto the Iwahori-Hecke algebra that sends $T_{h} \mapsto 1$ for all $h \in T$. "Setting $T_{h}=1$ " in the Yokonuma algebra relations recovers relations for the Iwahori-Hecke algebra,

$$
T_{i}^{2}=q^{-1}+q^{-1}(q-1) T_{i}, \quad \underbrace{T_{i} T_{j} \cdots}_{m_{i j} \text { terms }}=\underbrace{T_{j} T_{i} \cdots}_{m_{i j} \text { terms }} .
$$

Furthermore, there is a surjective map from the Iwahori Hecke algebra onto the group algebra of the Weyl group given by $T_{i} \mapsto s_{i}$ and $q \mapsto 1$. Thus, by "setting $T_{i}=s_{i}$ and $q=1$ " we retrieve the Coxeter relations of $W$,

$$
s_{i}^{2}=1, \quad \underbrace{s_{i} s_{j} s_{i} \cdots}_{m_{i j} \text { terms }}=\underbrace{s_{j} s_{i} s_{j} \cdots}_{m_{i j} \text { terms }} .
$$

2. The Gelfand-Graev Hecke algebra. By definition, if $\mu_{\alpha} \neq 0$ for all simple roots $\alpha$, then $\psi_{\mu}$ is in general position. The Gelfand-Graev Hecke algebra $\mathcal{H}_{\mu}$ is commutative ([Yo1, St, Theorem 49]).

\section{Parabolic subalgebras of $\mathcal{H}_{\mu}$}

Let $\psi_{\mu}: U \rightarrow G$ be as in (2.10). Fix a subset $J \subseteq\left\{\alpha_{1}, \alpha_{2}, \ldots, \alpha_{\ell}\right\}$ such that

$$
J \supseteq\left\{\alpha_{i} \text { simple root } \mid \mu_{\alpha_{i}} \neq 0\right\} .
$$

For example, if $\psi_{\mu}$ is in general position, then $J=\left\{\alpha_{1}, \alpha_{2}, \ldots, \alpha_{\ell}\right\}$, but if $\psi_{\mu}$ is trivial, then $J$ could be any subset.

Let

$$
W_{J}=\left\langle s_{i} \in W \quad \mid \alpha_{i} \in J\right\rangle, \quad P_{J}=\left\langle U, T, W_{J}\right\rangle, \quad \text { and } \quad R_{J}=\mathbb{Z}-\operatorname{span}\left\{\alpha_{i} \in J\right\} \cap R .
$$

Then $P_{J}$ has subgroups

$$
L_{J}=\left\langle T, W_{J}, U_{\alpha} \mid \alpha \in R_{J}\right\rangle \text { and } U_{J}=\left\langle U_{\alpha} \mid \alpha \in R^{+}-R_{J}\right\rangle
$$

(a Levi subgroup and the unipotent radical of $P_{J}$, respectively). Note that

$$
U_{J} L_{J}=P_{J}, \quad U_{J} \cap L_{J}=1, \quad \text { and, in fact, } \quad P_{J}=U_{J} \rtimes L_{J} .
$$

Define the idempotents of $\mathbb{C} U$,

$$
e_{\mu J}=\frac{1}{\left|L_{J} \cap U\right|} \sum_{u \in L_{J} \cap U} \psi_{\mu}\left(u^{-1}\right) u \quad \text { and } \quad e_{J}^{\prime}=\frac{1}{\left|U_{J}\right|} \sum_{u \in U_{J}} u,
$$

so that $e_{\mu}=e_{\mu J} e_{J}^{\prime}$ is the decomposition of $e_{\mu}$ with respect to $P=L_{J} U_{J}$. 
The group homomorphisms

$$
\begin{aligned}
& P_{J} \longrightarrow L_{J} \\
& l u
\end{aligned} \quad \text { and } \quad \begin{aligned}
& P_{J} \\
& l u
\end{aligned} \quad \begin{aligned}
& G \\
& l u
\end{aligned} \quad \text { for } l \in L_{J}, u \in U_{J},
$$

induce functors

$$
\begin{array}{ccc}
\operatorname{Inf}_{L_{J}}^{P_{J}}:\left\{L_{J} \text {-modules }\right\} & \longrightarrow & \left\{P_{J} \text {-modules }\right\} \\
M & \mapsto & e_{J}^{\prime} M
\end{array}
$$

and

$$
\begin{array}{ccc}
\operatorname{Ind}_{P_{J}}^{G}:\left\{P_{J} \text {-modules }\right\} & \longrightarrow & \{G \text {-modules }\} \\
M^{\prime} & \mapsto \mathbb{C} G \otimes_{\mathbb{C} P_{J}} M^{\prime}
\end{array}
$$

whose composition is the functor $\operatorname{Indf}_{L_{J}}^{G}$. In the special case when $\left(\mathbb{C} L_{J}\right) e$ is an $L_{J}$-module with corresponding idempotent $e$,

$$
\begin{array}{ccc}
\operatorname{Indf}_{L_{J}}^{G}:\left\{L_{J} \text {-modules }\right\} & \longrightarrow & \{G \text {-modules }\} \\
\mathbb{C} L_{J} e & \mapsto & \mathbb{C} G e e_{J}^{\prime} .
\end{array}
$$

The map $\psi_{\mu}: U \rightarrow \mathbb{C}^{*}$ restricts to a linear character $\operatorname{Res}_{U \cap L_{J}}^{U}\left(\psi_{\mu}\right): L_{J} \cap U \rightarrow \mathbb{C}^{*}$. To make the notation less heavy-handed, write $\psi_{\mu}: L_{J} \cap U \rightarrow \mathbb{C}^{*}$, for $\operatorname{Res}_{U \cap L_{J}}^{U}\left(\psi_{\mu}\right)$.

Lemma 3.1. Let $\psi_{\mu}$ be as in (2.10). Then

$$
\operatorname{Ind}_{U}^{G}\left(\psi_{\mu}\right) \cong \operatorname{Indf}_{L_{J}}^{G}\left(\operatorname{Ind}_{U \cap L_{J}}^{L_{J}}\left(\psi_{\mu}\right)\right) .
$$

Proof. Recall $\operatorname{Ind}_{U}^{G}\left(\psi_{\mu}\right) \cong \mathbb{C} G e_{\mu}$. On the other hand,

$$
\operatorname{Ind}_{U \cap L_{J}}^{L_{J}}\left(\psi_{\mu}\right) \cong \mathbb{C} L_{J} e_{\mu J} \quad \text { implies } \quad \operatorname{Indf}_{L_{J}}^{G}\left(\operatorname{Ind}_{U \cap L_{J}}^{L_{J}}\left(\psi_{\mu}\right)\right) \cong \mathbb{C} G e_{\mu J} e_{J}^{\prime},
$$

where $e_{\mu J}$ is as in (3.3). But $e_{\mu J} e_{J}^{\prime}=e_{\mu}$, so

$$
\operatorname{Ind}_{U}^{G}\left(\psi_{\mu}\right) \cong \mathbb{C} G e_{\mu} \cong \mathbb{C} e_{\mu J} e_{J}^{\prime} \cong \operatorname{Indf}_{L_{J}}^{G}\left(\operatorname{Ind}_{U \cap L_{J}}^{L_{J}}\left(\psi_{\mu}\right)\right) .
$$

Theorem 3.1. The map

$$
\begin{aligned}
\theta: \operatorname{End}_{\mathbb{C} L_{J}}\left(\operatorname{Ind}_{U \cap L_{J}}^{L_{J}}\left(\psi_{\mu}\right)\right) & \longrightarrow \mathcal{H}_{\mu} \\
e_{\mu J} v e_{\mu J} & \mapsto e_{\mu} v e_{\mu}, \quad \text { for } v \in L_{J} \cap N_{\mu},
\end{aligned}
$$

is an injective algebra homomorphism.

Proof. Since $L_{J}$ normalizes $U_{J}$ and $e_{J}^{\prime} e_{\mu J}=e_{\mu}$,

$$
e_{\mu} v e_{\mu}=e_{J}^{\prime} e_{\mu J} v e_{J}^{\prime} e_{\mu J}=e_{J}^{\prime} e_{\mu J} v e_{\mu J}
$$

so the map $\theta$ is given by multiplying $e_{\mu J} v e_{\mu J}$ on the left by $e_{J}^{\prime}$. Thus, $\theta$ is well defined and injective. Because $e_{J}^{\prime}$ commutes with $e_{\mu J} v e_{\mu J}$ for $v \in L_{J}, \theta$ is also a homomorphism.

Write

$$
\mathcal{L}_{J}=\theta\left(\operatorname{End}_{\mathbb{C} L_{J}}\left(\operatorname{Ind}_{U \cap L_{J}}^{L_{J}}\left(\psi_{\mu}\right)\right)\right) \subseteq \mathcal{H}_{\mu} .
$$

The $\mathcal{L}_{J}$ are "parabolic" subalgebras of $\mathcal{H}_{\mu}$, in that they have a similar role in the representation theory of $\mathcal{H}_{\mu}$ as parabolic subgroups $P_{J}$ have in the representation theory of $G$. 
3.1. Weight space decompositions for $\mathcal{H}_{\mu}$-modules. An important special case of Theorem 3.1 is when

$$
J=J_{\mu}=\left\{\alpha_{i} \text { simple root } \mid \mu_{\alpha_{i}} \neq 0\right\},
$$

so that $J_{\mu}$ is minimal satisfying (3.1). Write $L_{\mu}=L_{J_{\mu}}, W_{\mu}=W_{J_{\mu}}$, etc.

Corollary 1. The algebra $\mathcal{L}_{\mu}$ is a nonzero commutative subalgebra of $\mathcal{H}_{\mu}$.

Proof. As a character of $U \cap L_{\mu}, \psi_{\mu}$ is in general position, so $\operatorname{Ind}_{L_{\mu} \cap U}^{L_{\mu}}\left(\psi_{\mu}\right)$ is a Gelfand-Graev module and $\mathcal{L}_{\mu}$ is a Gelfand-Graev Hecke algebra (see Example 2 in Section 2.3).

Since $\mathcal{L}_{\mu}$ is commutative, all the irreducible $\mathcal{L}_{\mu}$-modules are one-dimensional. Let $\hat{\mathcal{L}}_{\mu}$ be an indexing set for the irreducible modules of $\mathcal{L}_{\mu}$. Suppose $V$ is an $\mathcal{H}_{\mu}$-module. Since $\mathcal{L}_{\mu} \cong \operatorname{End}_{\mathbb{C} L_{\mu}}\left(\operatorname{Ind}_{U \cap L_{\mu}}^{L_{\mu}}\left(\psi_{\mu}\right)\right), \mathcal{L}_{\mu}$ is semisimple, and as an $\mathcal{L}_{\mu^{-}}$ module,

$$
V \cong \bigoplus_{\gamma \in \hat{\mathcal{L}}_{\mu}} V_{\gamma}, \quad \text { where } \quad V_{\gamma}=\left\{v \in V \mid x v=\gamma(x) v, x \in \mathcal{L}_{\mu}\right\} .
$$

If $\gamma \in \hat{\mathcal{L}}_{\mu}$, then $V_{\gamma}$ is the $\gamma$-weight space of $V$, and $\gamma$ is a weight of $V$ if $V_{\gamma} \neq 0$.

\section{Examples.}

1. In the Yokonuma algebra $\psi_{\mu}=\mathbb{1}, J_{\mathbb{1}}=\emptyset$ and $\mathcal{L}_{\mathbb{1}}=e_{\mathbb{1}} \mathbb{C} T e_{\mathbb{1}} \cong \mathbb{C} T$.

2. In the Gelfand-Graev Hecke algebra case, $J_{\mu}=\left\{\alpha_{1}, \alpha_{2}, \ldots, \alpha_{\ell}\right\}$ and $\mathcal{L}_{\mu}=$ $\mathcal{H}_{\mu}$.

Remark. Since $\operatorname{dim}\left(V_{\gamma}\right)$ can be greater than one, $\mathcal{L}_{\mu}$ is not in general a maximal commutative subalgebra of $\mathcal{H}_{\mu}$.

\section{Multiplication OF BASIS ELEMENTS}

This section examines the decomposition of products in terms of the natural basis

$$
\left(e_{\mu} u e_{\mu}\right)\left(e_{\mu} v e_{\mu}\right)=\sum_{v^{\prime} \in N_{\mu}} c_{u v}^{v^{\prime}}\left(e_{\mu} v^{\prime} e_{\mu}\right) .
$$

In particular, Theorem 4.1 below, gives a set of braid-like relations (similar to those of the Yokonuma algebra) for manipulating the products, and Corollary 3 gives a recursive formula for computing these products.

4.1. Chevalley group relations. The relations governing the interaction between the subgroups $N, U$, and $T$ will be critical in describing the Hecke algebra multiplication in the following section. They can all be found in [St, §3].

The subgroup

$$
U=\left\langle x_{\alpha}(t) \mid \alpha \in R^{+}, t \in \mathbb{F}_{q}\right\rangle
$$

has generators $\left\{x_{\alpha}(t) \mid \alpha \in R^{+}, t \in \mathbb{F}_{q}\right\}$, with relations

$$
\begin{gathered}
x_{\alpha}(a) x_{\beta}(b) x_{\alpha}(a)^{-1} x_{\beta}(b)^{-1}=\prod_{\substack{\gamma=i \alpha+j \beta \in R^{+} \\
i, j \in \mathbb{Z}>0}} x_{\gamma}\left(z_{i j}(\alpha, \beta) a^{i} b^{j}\right), \\
x_{\alpha}(a) x_{\alpha}(b)=x_{\alpha}(a+b),
\end{gathered}
$$


where $z_{i j}(\alpha, \beta) \in \mathbb{Z}$ depends on $i, j, \alpha, \beta$ and a fixed order on the positive roots $R^{+}$, but not on $a, b \in \mathbb{F}_{q}\left[\mathrm{St}\right.$, Lemma 15]. The $z_{i j}(\alpha, \beta)$ have been explicitly computed for various types in $[\mathrm{De}, \mathrm{St}$.

The subgroup $N$ has generators $\left\{w_{i}(1), h_{H}(t) \mid i=1,2, \ldots, \ell, H \in \mathfrak{h}_{\mathbb{Z}}, t \in \mathbb{F}_{q}^{*}\right\}$, with relations

$$
\begin{aligned}
& w_{i}(1)^{2}=h_{i}(-1), \\
& \text { (N2) } \quad \underbrace{w_{i}(1) w_{j}(1) w_{i}(1) w_{j}(1) \cdots}_{m_{i j} \text { terms }}=\underbrace{w_{j}(1) w_{i}(1) w_{j}(1) w_{i}(1) \cdots}_{m_{i j} \text { terms }}, \\
& \text { where }\left(s_{i} s_{j}\right)^{m_{i j}}=1 \text { in } W, \\
& w_{i}(1) h_{H}(t)=h_{s_{i}(H)}(t) w_{i}(1), \\
& h_{H}(a) h_{H}(b)=h_{H}(a b), \\
& \text { (N3) } h_{H}(a) h_{H^{\prime}}(b)=h_{H^{\prime}}(b) h_{H}(a), \quad \text { for } H, H^{\prime} \in \mathfrak{h}, \\
& \text { (N5) } h_{H}(a) h_{H^{\prime}}(a)=h_{H+H^{\prime}}(a), \quad \text { for } H, H^{\prime} \in \mathfrak{h}, \\
& \text { (N6) } \text { (N7) } h_{H_{1}}\left(t_{1}\right) h_{H_{2}}\left(t_{2}\right) \cdots h_{H_{k}}\left(t_{k}\right)=1, \text { if } t_{1}^{\lambda_{j}\left(H_{1}\right)} \cdots t_{k}^{\lambda_{j}\left(H_{k}\right)}=1 \text { for all } 1 \leq j \leq r,
\end{aligned}
$$

where $\lambda_{j}: \mathfrak{h} \rightarrow \mathbb{C}$ depends on $V$ as in (2.4).

The double-coset decomposition of $G$ (2.13) implies $G=\langle U, N\rangle$. Thus, $G$ is generated by $\left\{x_{\alpha}(a), w_{i}(1), h_{H}(b) \mid \alpha \in R^{+}, a \in \mathbb{F}_{q}, i=1,2, \ldots, \ell, H \in \mathfrak{h}_{\mathbb{Z}}, b \in \mathbb{F}_{q}^{*}\right\}$ with relations (U1)-(N7) and

(UN1) $w_{i}(1) x_{\alpha}(t) w_{i}(1)^{-1}=x_{s_{i}(\alpha)}\left(c_{i \alpha} t\right)$, for $\alpha \neq \alpha_{i}$, where $c_{i \alpha}= \pm 1$,

$$
\begin{aligned}
h x_{\alpha}(b) h^{-1} & =x_{\alpha}(\alpha(h) b), \quad \text { for } h \in T, \\
w_{i}(1) x_{i}(t) w_{i}(1) & =x_{i}\left(-t^{-1}\right) h_{i}\left(-t^{-1}\right) w_{i}(1) x_{i}\left(-t^{-1}\right), \\
& \text { where } x_{i}(t)=x_{\alpha_{i}}(t) \text { and } t \neq 0,
\end{aligned}
$$

where for $\alpha \in R$ and $h_{H}(t) \in T$,

$$
\alpha\left(h_{H}(t)\right)=t^{\alpha(H)} .
$$

Note that relation (UN3) is not conjugation by $w_{i}(1)$.

Fix a $\psi_{\mu}: U \rightarrow \mathbb{C}^{*}$ as in (2.10). For $k \in \mathbb{F}_{q}$, let

$$
e_{\alpha}(k)=\frac{1}{q} \sum_{t \in \mathbb{F}_{q}} \psi\left(-\mu_{\alpha} k t\right) x_{\alpha}(t) \quad \text { with the convention } \quad e_{\alpha}=e_{\alpha}(1) .
$$

Note that for any given ordering of the positive roots, the decomposition

$$
U=\prod_{\alpha \in R^{+}} U_{\alpha} \quad \text { implies } \quad e_{\mu}=\prod_{\alpha \in R^{+}} e_{\alpha} .
$$

In particular, given any $\alpha \in R^{+}$, we may choose the ordering of the positive roots to have $e_{\alpha}$ appear either first or last. Therefore, since $e_{\alpha}$ is an idempotent,

$$
e_{\mu} e_{\alpha}=e_{\mu}=e_{\alpha} e_{\mu}
$$

If $w=s_{i_{1}} s_{i_{2}} \cdots s_{i_{r}} \in W$ with $r$ minimal, then let

$$
R_{w}=\left\{\alpha \in R^{+} \mid w(\alpha) \in R^{-}\right\}=\left\{\alpha_{i_{r}}, s_{i_{r}}\left(\alpha_{i_{r-1}}\right), \ldots, s_{i_{r}} s_{i_{r-1}} \cdots s_{i_{2}}\left(\alpha_{i_{1}}\right)\right\}
$$

where the second equality is from [Bo, VI.1, Corollary 2 of Proposition 17]. 
Lemma 4.1. Let $v \in N, w=\pi(v)$ (with $\pi: N \rightarrow W$ as in (2.6) ), and for $\alpha \in R^{+}$, let $v x_{\alpha}(t) v^{-1}=x_{w \alpha}\left(c_{v \alpha} t\right)$, with $c_{v \alpha}= \pm 1$ as in (UN1). Then

$$
\begin{aligned}
v e_{\alpha}(k) v^{-1} & =e_{w \alpha}\left(\mu_{\alpha} \mu_{w \alpha}^{-1} c_{v \alpha} k\right), & & \text { if } \alpha \notin R_{w}, \\
v e_{\alpha} v^{-1} & =e_{w \alpha}, & & \text { if } \alpha \notin R_{w}, v \in N_{\mu}, \\
h e_{\alpha}(k) h^{-1} & =e_{\alpha}\left(k \alpha(h)^{-1}\right), & & \text { for } h \in T, \\
e_{\mu} x_{\alpha}(t) & =\psi\left(\mu_{\alpha} t\right) e_{\mu}=x_{\alpha}(t) e_{\mu}, & & \text { for } \alpha \in R^{+} .
\end{aligned}
$$

Proof. (E1) Using relation (UN1),

$$
\begin{aligned}
w e_{\alpha}(k) w^{-1} & =\frac{1}{q} \sum_{t \in \mathbb{F}_{q}} \psi\left(-\mu_{\alpha} k t\right) w x_{\alpha}(t) w^{-1}=\frac{1}{q} \sum_{t \in \mathbb{F}_{q}} \psi\left(-\mu_{\alpha} k t\right) x_{w \alpha}\left(c_{v \alpha} t\right) \\
& =\frac{1}{q} \sum_{t^{\prime} \in \mathbb{F}_{q}} \psi\left(-\mu_{\alpha} c_{v \alpha} k t^{\prime}\right) x_{w \alpha}\left(t^{\prime}\right)=e_{w \alpha}\left(\mu_{\alpha} \mu_{w \alpha}^{-1} c_{v \alpha} k\right) .
\end{aligned}
$$

(E2) Suppose $\alpha \notin R_{w}$. Since $v \in N_{\mu}$,

$$
\begin{aligned}
\psi\left(\mu_{\alpha} t\right)=\psi_{\mu}\left(x_{\alpha}(t)\right)=\psi_{\mu}\left(v x_{\alpha}(t) v^{-1}\right) & =\psi_{\mu}\left(x_{w \alpha}(k t)\right) & & (\text { by }(\text { UN1) }) \\
& =\psi\left(\mu_{w \alpha} k t\right), & & \text { for some } k \in \mathbb{Z}_{\neq 0} .
\end{aligned}
$$

In particular, since $\psi$ is nontrivial, $\mu_{\alpha}=k \mu_{w \alpha}$. Thus,

$$
v e_{\alpha} v^{-1}=\frac{1}{q} \sum_{t \in \mathbb{F}_{q}} \psi\left(-\mu_{\alpha} t\right) x_{w \alpha}(k t)=\frac{1}{q} \sum_{t^{\prime} \in \mathbb{F}_{q}} \psi\left(-\mu_{\alpha} k^{-1} t^{\prime}\right) x_{w \alpha}\left(t^{\prime}\right)=e_{w \alpha} .
$$

(E3) Since $h x_{\alpha}(t) h^{-1}=x_{\alpha}(\alpha(h) t)$,

$$
\begin{aligned}
h e_{\alpha}(k) h^{-1} & =\frac{1}{q} \sum_{t \in \mathbb{F}_{q}} \psi\left(-\mu_{\alpha} k t\right) x_{\alpha}(\alpha(h) t) \\
& =\sum_{t \in \mathbb{F}_{q}} \psi\left(-\mu_{\alpha} k t \alpha(h)^{-1}\right) x_{\alpha}(t)=e_{\alpha}\left(k \alpha(h)^{-1}\right) .
\end{aligned}
$$

(E4) The element $e_{\alpha}$ is the minimal central idempotent of $\mathbb{C} U_{\alpha}$ that corresponds to the character $x_{\alpha}(t) \mapsto \psi\left(\mu_{\alpha} t\right)$. Therefore, by (4.4), $e_{\mu} x_{\alpha}(t)=e_{\mu} e_{\alpha} x_{\alpha}(t)=$ $\psi\left(\mu_{\alpha} t\right) e_{\mu}$.

4.2. Local Hecke algebra relations. Let $u=u_{1} u_{2} \cdots u_{r} u_{T} \in N$ according to a minimal expression $s_{i_{1}} s_{i_{2}} \cdots s_{i_{r}} \in W$ (see (2.8)). For $1 \leq k \leq r$ define constants $c_{k}= \pm 1$ and roots $\beta_{k} \in R^{+}$by the equation

$$
x_{\beta_{k}}\left(c_{k} t\right)=\left(u_{k+1} \cdots u_{r}\right)^{-1} x_{\alpha_{i_{k}}}(t)\left(u_{k+1} \cdots u_{r}\right) .
$$

Note that $R_{\pi(u)}=\left\{\beta_{1}, \beta_{2}, \ldots, \beta_{r}\right\}$ (see (4.5)). Define $f_{u} \in \mathbb{F}_{q}\left[y_{1}, y_{2}, \ldots, y_{r}\right]$ by

$$
f_{u}=-\frac{\mu_{\beta_{1}} c_{1}}{\beta_{1}\left(u_{T}\right)} y_{1}-\frac{\mu_{\beta_{2}} c_{2}}{\beta_{2}\left(u_{T}\right)} y_{2}-\cdots-\frac{\mu_{\beta_{r}} c_{r}}{\beta_{r}\left(u_{T}\right)} y_{r},
$$

and for $k=1,2, \ldots, r$ write

$$
u_{k}(t)=w_{i_{k}}(1) x_{i_{k}}(t) .
$$

In the following theorem we evaluate polynomials $f \in \mathbb{F}_{q}\left[y_{1}, \ldots, y_{r}\right]$ at points in $t=\left(t_{1}, \ldots, t_{r}\right) \in \mathbb{F}_{q}^{r}$ by $f(t)=f\left(t_{1}, \ldots, t_{r}\right)$, where $y_{j}(t)=t_{j}$ for $1 \leq j \leq r$. 
Theorem 4.1. Let $u=u_{1} u_{2} \cdots u_{r} u_{T}, v=v_{1} v_{2} \cdots v_{s} v_{T} \in N_{\mu}$ according to minimal expressions $s_{i_{1}} s_{i_{2}} \cdots s_{i_{r}} \in W$ and $s_{j_{1}} s_{j_{2}} \cdots s_{j_{s}} \in W$, respectively, as in (2.8). Then

(a)

$$
\left(e_{\mu} u e_{\mu}\right)\left(e_{\mu} v e_{\mu}\right)=\frac{1}{q^{r}} \sum_{t \in \mathbb{F}_{q}^{r}}\left(\psi \circ f_{u}\right)(t) e_{\mu}\left(u_{1}\left(t_{1}\right) u_{2}\left(t_{2}\right) \cdots u_{r}\left(t_{r}\right)\right)\left(v_{1} v_{2} \cdots v_{s}\right) h e_{\mu},
$$

where $h=v_{T} v^{-1} u_{T} v \in T$.

(b) The following local relations suffice to compute the product $\left(e_{\mu} u e_{\mu}\right)\left(e_{\mu} v e_{\mu}\right)$.

$\left(w_{i}(1) x_{i}(a)\right) x_{\alpha}(b)=\prod_{\substack{\gamma=m \alpha_{i}+n \alpha \in R^{+} \\ m \geq 0, n>0}} x_{s_{i} \gamma}\left(c_{i \gamma} z_{m n}\left(\alpha_{i}, \alpha\right) a^{m} b^{n}\right)\left(w_{i}(1) x_{i}(a)\right), \quad$ for $\alpha \neq \alpha_{i}$,

$$
\begin{aligned}
\left(w_{i}(1) x_{i}(a)\right) x_{i}(b) & =\left(w_{i}(1) x_{i}(a+b)\right), \\
h_{\alpha}(a) h_{\alpha}(b) & =h_{\alpha}(a b), \\
h_{\alpha}(a) h_{\beta}(b) & =h_{\beta}(b) h_{\alpha}(a), \\
w_{i}(1)^{2} & =h_{i}(-1), \\
\underbrace{w_{i}(1) w_{j}(1) w_{i}(1) w_{j}(1) \cdots}_{m_{i j} \text { terms }} & =\underbrace{w_{j}(1) w_{i}(1) w_{j}(1) w_{i}(1) \cdots}_{m_{i j} \text { terms }},
\end{aligned}
$$

where $f, g \in \mathbb{F}_{q}\left[y_{1}^{ \pm 1}, \ldots, y_{r}^{ \pm 1}\right], t \in \mathbb{F}_{q}^{r}, \alpha, \beta \in R^{+}, 1 \leq i \leq \ell, z_{0,1}\left(\alpha_{i}, \alpha\right)=1$, and $m_{i j}$ is the order of $s_{i} s_{j}$ in $W$.

Proof. (a) Order the positive roots so that by (4.3)

$$
\begin{aligned}
& e_{\mu} u e_{\mu} v e_{\mu}=e_{\mu} u\left(\prod_{\alpha \notin R_{\pi(u)}} e_{\alpha}\right) e_{\beta_{1}} e_{\beta_{2}} \cdots e_{\beta_{r}} v e_{\mu} \\
& =e_{\mu}\left(\prod_{\alpha \notin R_{\pi(u)}} e_{\pi(u) \alpha}\right) u e_{\beta_{1}} e_{\beta_{2}} \cdots e_{\beta_{r}} v e_{\mu} \\
& =e_{\mu} u e_{\beta_{1}} e_{\beta_{2}} \cdots e_{\beta_{r}} v e_{\mu} \\
& =e_{\mu} u_{1} u_{2} \cdots u_{r} u_{T} e_{\beta_{1}} e_{\beta_{2}} \cdots e_{\beta_{r}} v e_{\mu} \\
& =e_{\mu} u_{1} u_{2} \cdots u_{r} e_{\beta_{1}}\left(\frac{1}{\beta_{1}\left(u_{T}\right)}\right) e_{\beta_{2}}\left(\frac{1}{\beta_{2}\left(u_{T}\right)}\right) \\
& \quad \cdots e_{\beta_{r}}\left(\frac{1}{\beta_{r}\left(u_{T}\right)}\right) u_{T} v e_{\mu}
\end{aligned}
$$




$$
\begin{aligned}
& =e_{\mu} u_{1} e_{\alpha_{i_{1}}}\left(\frac{\mu_{\beta_{1} c_{1}}}{\mu_{\alpha_{i} \beta_{1}\left(u_{T}\right)}}\right) u_{2} e_{\alpha_{i_{2}}}\left(\frac{\mu_{\beta_{2}} c_{2}}{\mu_{\alpha_{i_{2}} \beta_{2}\left(u_{T}\right)}}\right) \\
& \cdots u_{r} e_{\alpha_{i_{r}}}\left(\frac{\mu_{\beta_{r}} c_{r}}{\mu_{\alpha_{i_{r}}} \beta_{r}\left(u_{T}\right)}\right) u_{T} v e_{\mu} \\
& =e_{\mu} u_{1} e_{\alpha_{i_{1}}}\left(\frac{\mu_{\beta_{1}} c_{1}}{\mu_{\alpha_{i_{1}} \beta_{1}\left(u_{T}\right)}}\right) u_{2} e_{\alpha_{i_{2}}}\left(\frac{\mu_{\beta_{2}} c_{2}}{\mu_{\alpha_{i_{2}} \beta_{2}\left(u_{T}\right)}}\right) \\
& \cdots u_{r} e_{\alpha_{i_{r}}}\left(\frac{\mu_{\beta_{r}} c_{r}}{\mu_{\alpha_{i_{r}} \beta_{r}\left(u_{T}\right)}}\right) v_{1} \cdots v_{s} v_{T} v^{-1} u_{T} v e_{\mu} \\
& =\frac{e_{\mu}}{q^{r}} \sum_{t_{1}, \ldots, t_{r} \in \mathbb{F}_{q}} \psi\left(-\frac{\mu_{\beta_{1} c_{1} t_{1}}}{\beta_{1}\left(u_{T}\right)}\right) u_{1}\left(t_{1}\right) \cdots \psi\left(-\frac{\mu_{\beta_{r}} c_{r} t_{r}}{\beta_{r}\left(u_{T}\right)}\right) u_{r}\left(t_{r}\right) \\
& \times v_{1} \cdots v_{s} h e_{\mu} \quad \text { (definition of } e_{\alpha}, u_{\alpha}(t) \text { ) } \\
& =\frac{1}{q^{r}} \sum_{t \in \mathbb{F}_{q}^{r}}\left(\psi \circ f_{u}\right)(t) e_{\mu} u_{1}\left(t_{1}\right) \cdots u_{r}\left(t_{r}\right) v_{1} \cdots v_{s} h e_{\mu}
\end{aligned}
$$

(by $(\mathcal{H}(5)$ ),

where $h=v_{T} v^{-1} u_{T} v \in T$, as desired.

(b) First, note that these relations are in fact correct (though not necessarily

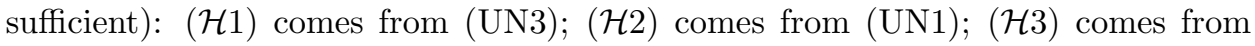

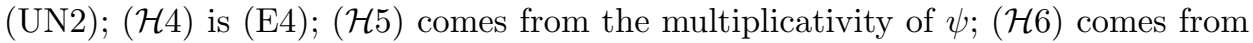

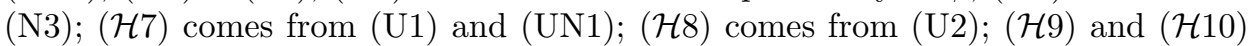

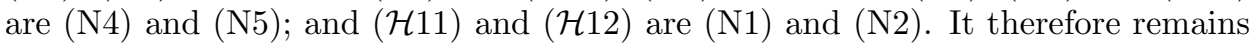
to show sufficiency.

By (a) we may write

$$
\left(e_{\mu} u e_{\mu}\right)\left(e_{\mu} v e_{\mu}\right)=\frac{1}{q^{r}} \sum_{t \in \mathbb{F}_{q}^{r}}(\psi \circ f)(t) e_{\mu} u_{1}\left(t_{1}\right) \cdots u_{r}\left(t_{r}\right) v_{1} \cdots v_{s} h e_{\mu}
$$

for some $f \in \mathbb{F}_{q}\left[y_{1}, \ldots, y_{r}\right]$ and $h \in T$. Say $t_{k}$ is resolved if the only parts of the summands depending on $t_{k}$ are $(\psi \circ f)$ and $h$. The product is reduced when all the $t_{k}$ are resolved. We will show how to resolve $t_{r}$ and the result will follow by induction.

Use relation (H2) to define the constant $d$ and the root $\gamma \in R$ by

$$
\left(v_{1} v_{2} \cdots v_{s}\right)^{-1} x_{\alpha_{i_{r}}}(t)\left(v_{1} v_{2} \cdots v_{s}\right)=x_{\gamma}(d t) \quad(\text { where } \ell(\pi(v))=s) .
$$

Note that $\gamma=\pi(v)^{-1}\left(\alpha_{i_{r}}\right)$ and $d= \pm 1$. There are two possible situations:

Case 1. $\gamma \in R^{+}$,

Case 2. $\gamma \in R^{-}$.

In Case 1,

$$
\begin{aligned}
& \left(e_{\mu} u e_{\mu}\right)\left(e_{\mu} v e_{\mu}\right)=\frac{1}{q^{r}} \sum_{t \in \mathbb{F}_{q}^{r}}(\psi \circ f)(t) e_{\mu} u_{1}\left(t_{1}\right) \cdots u_{r}{\underline{x_{i_{r}}}}_{\left(t_{r}\right)} v_{1} \cdots v_{s} h e_{\mu} \quad \text { (by (a)) } \\
& \left.=\frac{1}{q^{r}} \sum_{t \in \mathbb{F}_{q}^{r}}(\psi \circ f)(t) e_{\mu} u_{1}\left(t_{1}\right) \cdots u_{r-1}\left(t_{r-1}\right) u_{r} v_{1} \cdots v_{s} x_{\gamma}\left(d t_{r}\right) h e_{\mu} \quad \text { (by } \stackrel{(\mathcal{H} 2}{\longrightarrow}\right) \\
& =\frac{1}{q^{r}} \sum_{t \in \mathbb{F}_{q}^{r}}(\psi \circ f)(t) e_{\mu} u_{1}\left(t_{1}\right) \cdots u_{r-1}\left(t_{r-1}\right) u_{r} v_{1} \\
& \cdots v_{s} h \underline{x_{\gamma}\left(d \gamma(h)^{-1} t_{r}\right) e_{\mu}}
\end{aligned}
$$




$$
\begin{aligned}
=\frac{1}{q^{r}} \sum_{t \in \mathbb{F}_{q}^{r}}(\psi \circ f)(t) e_{\mu} u_{1}\left(t_{1}\right) \cdots u_{r-1}\left(t_{r-1}\right) u_{r} v_{1} \\
\quad \cdots v_{s} h \underline{\underline{\psi\left(\mu_{\gamma} d \gamma(h)^{-1} t_{r}\right)} e_{\mu}} \\
=\frac{1}{q^{r}} \sum_{t \in \mathbb{F}_{q}^{r}}(\psi \circ f)(t) e_{\mu} u_{1}\left(t_{1}\right) \cdots u_{r-1}\left(t_{r-1}\right) u_{r} v_{1} \\
\quad \cdots v_{s} h \underbrace{}_{\left(\psi \circ \mu_{\gamma} d \gamma(h)^{-1} y_{r}\right)(t) e_{\mu}} \\
=\frac{1}{q^{r}} \sum_{t \in \mathbb{F}_{q}^{r}}(\psi \circ g)(t) e_{\mu} u_{1}\left(t_{1}\right) \cdots u_{r-1}\left(t_{r-1}\right) u_{r} v_{1} \cdots v_{s} h e_{\mu},
\end{aligned}
$$

where $g=f+\mu_{\gamma} d \gamma(h)^{-1} y_{r}$. We have resolved $t_{r}$ in Case 1. Furthermore, since $\gamma \in R^{+}, v_{1}^{\prime} v_{2}^{\prime} \cdots v_{s+1}^{\prime}=u_{r} v_{1} v_{2} \cdots v_{s}$ still corresponds to a minimal expression in $W$.

In Case 2, $\gamma \in R^{-}$, so we can no longer move $x_{i_{r}}\left(t_{r}\right)$ past the $v_{j}$. Instead,

$$
\begin{aligned}
& \left(e_{\mu} u e_{\mu}\right)\left(e_{\mu} v e_{\mu}\right) \\
& =\frac{e_{\mu}}{q^{r}} \sum_{t \in \mathbb{F}_{q}^{r}} \underline{(\psi \circ f)(t)} u_{1}\left(t_{1}\right) \cdots u_{r-1}\left(t_{r-1}\right) \\
& \times u_{r} x_{i_{r}}\left(t_{r}\right) u_{r} u_{r}^{-1} v_{1} \cdots v_{s} h e_{\mu} \\
& =\frac{e_{\mu}}{q^{r}} \sum_{t^{\prime} \in \mathbb{F}^{r-1}} u_{1}\left(t_{1}\right) \cdots u_{r-1}\left(t_{r-1}\right) \\
& \times \sum_{t_{r} \in \mathbb{F}_{q}}(\psi \circ f)\left(t^{\prime}, t_{r}\right) \underline{u_{r} x_{i_{r}}\left(t_{r}\right) u_{r}} u_{r}^{-1} v_{1} \cdots v_{s} h e_{\mu} \\
& =\frac{e_{\mu}}{q^{r}} \sum_{t^{\prime} \in \mathbb{F}_{q}^{r-1}} u_{1}\left(t_{1}\right) \cdots u_{r-1}\left(t_{r-1}\right)(\psi \circ f)\left(t^{\prime}, 0\right) \\
& \times \underset{h_{i_{r}}(-1)}{\longrightarrow} u_{r}^{-1} v_{1} \cdots v_{s} h e_{\mu} \\
& +\frac{e_{\mu}}{q_{t^{\prime} \in \mathbb{F}_{q}^{r-1}}} \sum_{1} u_{1}\left(t_{1}\right) \cdots u_{r-1}\left(t_{r-1}\right) \\
& \times \sum_{t_{r} \in \mathbb{F}_{q}^{*}}(\psi \circ f)\left(t^{\prime},-t_{r}^{-1}\right) x_{i_{r}}\left(t_{r}\right) h_{i_{r}}\left(t_{r}\right) \underline{u_{r} x_{i_{r}}\left(t_{r}\right) u_{r}^{-1}} v_{1} \cdots v_{s} h e_{\mu} \\
& =\frac{e_{\mu}}{q^{r}} \sum_{t^{\prime} \in \mathbb{F}_{q}^{r-1}} u_{1}\left(t_{1}\right) \cdots u_{r-1}\left(t_{r-1}\right)(\longleftarrow \circ f)\left(t^{\prime}, 0\right) \\
& \times u_{r}^{-1} v_{1} \cdots v_{s} h_{-\gamma}(-1) h e_{\mu} \\
& +\frac{e_{\mu}}{q_{t^{\prime} \in \mathbb{F}_{q}^{r-1}}^{r}} u_{1}\left(t_{1}\right) \cdots u_{r-1}\left(t_{r-1}\right) \\
& \times \sum_{t_{r} \in \mathbb{F}_{q}^{*}}(\psi \circ f)\left(t^{\prime},-t_{r}^{-1}\right) x_{i_{r}}\left(t_{r}\right) h_{i_{r}}\left(t_{r}\right) \stackrel{x_{-\alpha_{i_{r}}}\left(-t_{r}\right)}{\longrightarrow} v_{1} \cdots v_{s} h e_{\mu}
\end{aligned}
$$




$$
\begin{aligned}
& =\frac{e_{\mu}}{q^{r}} \sum_{t^{\prime} \in \mathbb{F}_{q}^{r-1}}(\psi \circ f)\left(t^{\prime}, 0\right) u_{1}\left(t_{1}\right) \cdots u_{r-1}\left(t_{r-1}\right) \\
& \quad \times u_{r}^{-1} v_{1} \cdots v_{s} h_{-\gamma}(-1) h e_{\mu} \\
& +\frac{e_{\mu}}{q^{r}} \sum_{t^{\prime} \in \mathbb{F}_{q}^{r-1}} u_{1}\left(t_{1}\right) \cdots u_{r-1}\left(t_{r-1}\right) \\
& \times \sum_{t_{r} \in \mathbb{F}_{q}^{*}}(\psi \circ g)\left(t^{\prime},-t_{r}^{-1}\right) x_{i_{r}}\left(t_{r}\right) \stackrel{h_{i_{r}}\left(t_{r}\right)}{\longrightarrow} v_{1} \cdots v_{s} h e_{\mu}
\end{aligned}
$$

(where $g=f+\mu_{-\gamma} d(-\gamma(h))^{-1} y_{r}^{-1}$ (same as in the analogous steps in Case 1))

$$
\begin{aligned}
& =\frac{e_{\mu}}{q^{r}} \sum_{t^{\prime} \in \mathbb{F}_{q}^{r-1}}(\psi \circ f)\left(t^{\prime}, 0\right) u_{1}\left(t_{1}\right) \cdots u_{r-1}\left(t_{r-1}\right) \\
& \times u_{r}^{-1} v_{1} \cdots v_{s} h_{-\gamma}(-1) h e_{\mu} \\
& +\frac{e_{\mu}}{q^{r}} \sum_{t^{\prime} \in \mathbb{F}_{q}^{r-1}} u_{1}\left(t_{1}\right) \cdots u_{r-1}\left(t_{r-1}\right) \\
& \times \sum_{t_{r} \in \mathbb{F}_{q}^{*}}(\psi \circ g)\left(t^{\prime},-t_{r}^{-1}\right) x_{i_{r}}\left(t_{r}\right) v_{1} \cdots v_{s} h_{-\gamma}\left(t_{r}^{-1}\right) h e_{\mu} \\
& =\frac{e_{\mu}}{q^{r}} \sum_{t^{\prime} \in \mathbb{F}_{q}^{r-1}}(\psi \circ f)\left(t^{\prime}, 0\right) u_{1}\left(t_{1}\right) \cdots u_{r-1}\left(t_{r-1}\right) \\
& \times u_{r}^{-1} v_{1} \cdots v_{s} h_{-\gamma}(-1) h e_{\mu} \\
& +\frac{e_{\mu}}{q^{r}} \sum_{\substack{t^{\prime} \in \mathbb{F}_{q}^{r-1} \\
t_{r} \in \mathbb{F}_{q}^{*}}}(\psi \circ \varphi(g))\left(t^{\prime},-t_{r}^{-1}\right) \\
& \times\left(\prod_{\beta \in R^{+}} x_{\beta}\left(a_{\beta}\left(t^{\prime}, t_{r}\right)\right)\right) u_{1}\left(t_{1}\right) \cdots u_{r-1}\left(t_{r-1}\right) v_{1} \cdots v_{s} h^{\prime} e_{\mu}
\end{aligned}
$$

(where $\varphi: \mathbb{F}_{q}\left[y_{1}, \ldots, y_{r}\right] \rightarrow \mathbb{F}_{q}\left[y_{1}, \ldots, y_{r}\right]$ catalogues the substitutions to $g$ due to

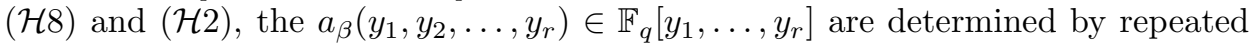

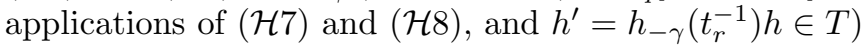

$$
\begin{aligned}
& =\frac{1}{q^{r}} \sum_{t^{\prime} \in \mathbb{F}_{q}^{r-1}}(\psi \circ f)\left(t^{\prime}, 0\right) e_{\mu} u_{1}\left(t_{1}\right) \cdots u_{r-1}\left(t_{r-1}\right) \\
& \times u_{r}^{-1} v_{1} \cdots v_{s} h_{-\gamma}(-1) h e_{\mu} \\
& +\frac{1}{q^{r}} \sum_{\substack{t^{\prime} \in \mathbb{F}^{r-1} \\
t_{r} \in \mathbb{F}_{q}^{*}}}(\psi \circ \varphi(g))\left(t^{\prime},-t_{r}^{-1}\right) e_{\mu} \\
& \times\left(\prod_{\beta \in R^{+}} \longleftarrow\left(\mu_{\beta} a_{\beta}\left(t^{\prime}, t_{r}\right)\right)\right) u_{1}\left(t_{1}\right) \cdots u_{r-1}\left(t_{r-1}\right) v_{1} \cdots v_{s} h^{\prime} e_{\mu}
\end{aligned}
$$




$$
\begin{gathered}
=\frac{1}{q^{r}} \sum_{t^{\prime} \in \mathbb{F}_{q}^{r-1}}(\psi \circ f)\left(t^{\prime}, 0\right) e_{\mu} u_{1}\left(t_{1}\right) \cdots u_{r-1}\left(t_{r-1}\right) \\
\quad \times u_{r}^{-1} v_{1} \cdots v_{s} h_{-\gamma}(-1) h e_{\mu} \\
+\frac{1}{q^{r}} \sum_{\substack{t^{\prime} \in \mathbb{F}_{q}^{r-1} \\
t_{r} \in \mathbb{F}_{q}^{*}}}\left(\psi \circ g_{2}\right)\left(t^{\prime},-t_{r}^{-1}\right) e_{\mu} u_{1}\left(t_{1}\right) \cdots u_{r-1}\left(t_{r-1}\right) \\
\times v_{1} \cdots v_{s} h^{\prime} e_{\mu}
\end{gathered}
$$

where $g_{2}=\varphi(g)+\sum_{\beta \in R^{+}} \mu_{\beta} a_{\beta}\left(y_{1}, \ldots, y_{r-1},-y_{r}^{-1}\right)$. In the first sum, use (H11) and (ㄱ12) to reduce $v_{1}^{\prime} \cdots v_{s-1}^{\prime}=u_{r}^{-1} v_{1} \cdots v_{s}$ into an expression that corresponds

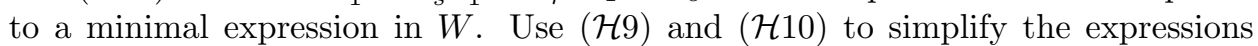
$h^{\prime}, h_{-\gamma}(-1) h \in T$. Now $t_{r}$ is resolved for Case 2, as desired.

Corollary 2 (Resolving $t_{k}$ ). Let $u=u_{1} u_{2} \cdots u_{k} \in N$ according to a minimal expression $s_{i_{1}} s_{i_{2}} \cdots s_{i_{k}} \in W\left(\right.$ with $\left.u_{T}=1\right)$. Suppose $v \in N$ and $f \in \mathbb{F}_{q}\left[y_{1}, y_{2}, \ldots, y_{k}\right]$. Define $\gamma \in R$ and $d \in \mathbb{C}$ by the equation $v^{-1} x_{i_{k}}(t) v=x_{\gamma}(d t)$. Then

Case 1. If $\ell\left(\pi\left(u_{k} v\right)\right)>\ell(\pi(v))$, then

$$
\begin{aligned}
\sum_{t \in \mathbb{F}_{q}^{k}} & (\psi \circ f)(t) e_{\mu} u_{1}\left(t_{1}\right) \cdots u_{k}\left(t_{k}\right) v e_{\mu} \\
& \left.=\sum_{t \in \mathbb{F}_{q}^{k}}\left(\psi \circ \underline{\left(f+\mu_{\gamma} d y_{k}\right.}\right)\right)(t) e_{\mu} u_{1}\left(t_{1}\right) \cdots u_{k-1}\left(t_{k-1}\right) \underline{u_{k} v} e_{\mu} .
\end{aligned}
$$

Case 2. If $\ell\left(\pi\left(u_{k} v\right)\right)<\ell(\pi(v))$, then

$$
\begin{aligned}
& \sum_{t \in \mathbb{F}_{q}^{k}}(\psi \circ f)(t) e_{\mu} u_{1}\left(t_{1}\right) \cdots u_{k}\left(t_{k}\right) v e_{\mu} \\
& =\sum_{\substack{t \in \mathbb{F}_{q}^{k} \\
t_{k}=0}}(\psi \circ f)(t) e_{\mu} u_{1}\left(t_{1}\right) \cdots u_{k-1}\left(t_{k-1}\right) \underline{u_{k} v} e_{\mu} \\
& \left.+\sum_{\substack{t \in \mathbb{F}_{q}^{k} \\
t_{k} \in \mathbb{F}_{q}^{*}}}\left(\psi \circ \underline{\left(\varphi_{k}(f)+\mu_{-\gamma} d y_{k}^{-1}\right.}\right)\right)(t) e_{\mu} u_{1}\left(t_{1}\right) \cdots u_{k-1}\left(t_{k-1}\right) \underline{h_{i_{k}}\left(-t_{k}\right) v e_{\mu}}, \\
& \text { where } \varphi_{k}: \mathbb{F}_{q}\left[y_{1}^{ \pm 1}, \ldots, y_{k}^{ \pm 1}\right] \rightarrow \mathbb{F}_{q}\left[y_{1}^{ \pm 1}, \ldots, y_{k}^{ \pm 1}\right] \text { is given by } \\
& \begin{aligned}
\sum_{\substack{t \in \mathbb{F}_{q}^{k} \\
t_{k} \in \mathbb{F}_{q}^{*}}}(\psi \circ f)(t) e_{\mu} u_{1}\left(t_{1}\right) \cdots u_{k-1}\left(t_{k-1}\right) & x_{i_{k}}\left(-t_{k}^{-1}\right) \\
= & \sum_{\substack{t \in \mathbb{F}_{q}^{k} \\
t_{k} \in \mathbb{F}_{q}^{*}}}\left(\psi \circ \underline{\varphi_{k}(f)}\right)(t) e_{\mu} u_{1}\left(t_{1}\right) \cdots u_{k-1}\left(t_{k-1}\right) .
\end{aligned}
\end{aligned}
$$

Proof. This corollary puts $v$ in the place of $u_{T} v$ in the proof of Theorem 4.1(b), and summarizes the steps taken in Case 1 and Case 2. The only slight adjustments are in Case 2: note that $u_{k} v=h_{i_{k}}(-1) u_{k}^{-1} v$ in the first summand, and there is a renormalization of $t_{k}$ in the second summand. 
4.3. Global Hecke algebra relations. Fix a decomposition $u=u_{1} u_{2} \cdots u_{r} u_{T} \in$ $N_{\mu}$ according a minimal expression $s_{i_{1}} s_{i_{2}} \cdots s_{i_{r}} \in W$ (see (2.8)). Suppose $v^{\prime} \in N_{\mu}$ and let $v=u_{T} v^{\prime}$.

For $0 \leq k \leq r$, let $\tau=\left(\tau_{1}, \tau_{2}, \ldots, \tau_{r-k}\right)$ be such that $\tau_{i} \in\{+0,-0,1\}$, where $+0,-0$, and 1 are symbols. If $\tau$ has $r-k$ elements, then the colength of $\tau$ is $\ell^{\vee}(\tau)=k$. For example, if $r=10$ and $\tau=(-0,1,+0,+0,1,1)$, then $\ell^{\vee}(\tau)=4$. For $i \in\{+0,-0,1\}$, let

$$
(i, \tau)=\left(i, \tau_{1}, \tau_{2}, \ldots, \tau_{r-k}\right) .
$$

By convention, if $\ell^{\vee}(\tau)=r$, then $\tau=\emptyset$.

Suppose $\ell^{\vee}(\tau)=k$. Define

$$
\Xi^{\tau}(u, v)=\frac{1}{q^{r}} \sum_{t \in \mathbb{F}_{q}^{\tau}}\left(\psi \circ f^{\tau}\right)(t) e_{\mu} u_{1}\left(t_{1}\right) \cdots u_{k}\left(t_{k}\right) v^{\tau}(t) e_{\mu},
$$

where

$$
\begin{gathered}
\mathbb{F}_{q}^{\tau}=\left\{\begin{array}{ll} 
& \text { if } \tau_{i-k}=+0, \text { then } t_{i} \in \mathbb{F}_{q} \\
t \in \mathbb{F}_{q}^{r} \mid \text { for } k<i \leq r, & \text { if } \tau_{i-k}=-0, \text { then } t_{i}=0 \\
& \text { if } \tau_{i-k}=1, \text { then } t_{i} \in \mathbb{F}_{q}^{*}
\end{array}\right\}, \\
v^{\tau}(t)=h_{i_{k+1}}\left(-t_{k+1}\right)^{\tau_{1}} u_{k+1}^{1-\tau_{1}} \cdots h_{i_{r}}\left(-t_{r}\right)^{\tau_{r-k}} u_{r}^{1-\tau_{r-k}} v,
\end{gathered}
$$

with $+0=-0=0 \in \mathbb{Z}$ and $1=1 \in \mathbb{Z}$ in (4.12); and $f^{\tau}$ is defined recursively by

$$
\begin{aligned}
f^{\emptyset}=f_{u} & =-\frac{\mu_{\beta_{1}} c_{1}}{\beta_{1}\left(u_{T}\right)} y_{1}-\frac{\mu_{\beta_{2}} c_{2}}{\beta_{2}\left(u_{T}\right)} y_{2}-\cdots-\frac{\mu_{\beta_{r}} c_{r}}{\beta_{r}\left(u_{T}\right)} y_{r} \quad \text { (as in (4.7)), } \\
f^{(i, \tau)} & = \begin{cases}f^{\tau}+\mu_{\gamma_{\tau}} d_{\tau} y_{k}, & \text { if } i=+0, \\
f^{\tau}, & \text { if } i=-0, \\
\varphi_{k}\left(f^{\tau}\right)+\mu_{-\gamma_{\tau}} d_{\tau} y_{k}^{-1}, & \text { if } i=1,\end{cases}
\end{aligned}
$$

where $\left(v^{\tau}\right)^{-1} x_{\alpha_{i_{k}}}(t) v^{\tau}=x_{\gamma_{\tau}}\left(d_{\tau} t\right)$ and the map $\varphi_{k}$ is as in Corollary 2, Case 2.

Remarks.

1. By (4.10) and Theorem 4.1(a), $\Xi^{\emptyset}(u, v)=\left(e_{\mu} u e_{\mu}\right)\left(e_{\mu} v^{\prime} e_{\mu}\right)$ (recall, $v=$ $\left.u_{T} v^{\prime}\right)$

2. If $\ell^{\vee}(\tau)=0$ so that $\tau$ is a string of length $r$, then

(a) $\Xi^{\tau}(u, v)=\frac{1}{q^{r}} \sum_{t \in \mathbb{F}_{q}^{\tau}}\left(\psi \circ f^{\tau}\right)(t) e_{\mu} v^{\tau}(t) e_{\mu}$ has no remaining factors of the form $u_{k}\left(t_{k}\right)$,

(b) $\Xi^{\tau}(u, v)=0$ unless $v^{\tau}(t) \in N_{\mu}$ for some $t \in \mathbb{F}_{q}$.

The following corollary gives relations for expanding $\Xi^{\tau}(u, v)$ (beginning with $\left.\Xi^{\emptyset}(u, v)\right)$ as a sum of terms of the form $\Xi^{\tau^{\prime}}$ with $\ell^{\vee}\left(\tau^{\prime}\right)=\ell^{\vee}(\tau)-1$. When each term has colength 0 (length $r$ ), then the product $\left(e_{\mu} u e_{\mu}\right)\left(e_{\mu} v^{\prime} e_{\mu}\right)$ is decomposed in terms of the basis elements of $\mathcal{H}_{\mu}$.

In summary, while we compute $f^{\tau}$ recursively by removing elements from $\tau$, we compute the product $\left(e_{\mu} u e_{\mu}\right)\left(e_{\mu} v^{\prime} e_{\mu}\right)$ by progressively adding elements to $\tau$.

Corollary 3 (The Global Alternative). Let $u, v^{\prime} \in N_{\mu}$ such that $u=u_{1} u_{2} \cdots u_{r} u_{T}$ decomposes according to a minimal expression in $W$. Let $v=u_{T} v^{\prime}$. Then

(a) $\left(e_{\mu} u e_{\mu}\right)\left(e_{\mu} v^{\prime} e_{\mu}\right)=\Xi^{\emptyset}(u, v)$. 
(b) If $\ell^{\vee}(\tau)=k$, then

$$
\Xi^{\tau}(u, v)= \begin{cases}\Xi^{(+0, \tau)}(u, v), & \text { if } \ell\left(\pi\left(u_{k} v^{\tau}\right)\right)>\ell\left(\pi\left(v^{\tau}\right)\right), \\ \Xi^{(-0, \tau)}(u, v)+\Xi^{(1, \tau)}(u, v), & \text { if } \ell\left(\pi\left(u_{k} v^{\tau}\right)\right)<\ell\left(\pi\left(v^{\tau}\right)\right) .\end{cases}
$$

Proof. (a) follows from Remark 1.

(b) Suppose $\ell^{\vee}(\tau)=k$. Note that

$$
\begin{aligned}
\Xi^{\tau}(u, v) & =\frac{1}{q^{r}} \sum_{t \in \mathbb{F}_{q}^{\tau}}\left(\psi \circ f^{\tau}\right)(t) e_{\mu} u_{1}\left(t_{1}\right) \cdots u_{k}\left(t_{k}\right) v^{\tau} e_{\mu} \\
& =\frac{1}{q^{r}} \sum_{t^{\prime \prime} \in\left(\mathbb{F}_{q}^{r-k}\right)^{\tau}} \sum_{t^{\prime} \in \mathbb{F}_{q}^{k}}\left(\psi \circ f^{\tau}\right)\left(t^{\prime}, t^{\prime \prime}\right) e_{\mu} u_{1}\left(t_{1}\right) \cdots u_{k}\left(t_{k}\right) v^{\tau} e_{\mu},
\end{aligned}
$$

where $\left(\mathbb{F}_{q}^{r-k}\right)^{\tau}=\left\{\left(t_{k+1}, \ldots, t_{r}\right) \in \mathbb{F}_{q}^{r-k} \quad \mid\right.$ restrictions according to $\left.\tau\right\}$ (as in (4.11)). Apply Corollary 2 to the inside sum with $f:=f^{\tau}, v:=v^{\tau}$. Note that the corollary relations imply

$$
\begin{aligned}
&\left\{t^{\prime} \in \mathbb{F}_{q}^{k}\right\} \text { becomes } \begin{cases}\left\{t^{\prime} \in \mathbb{F}_{q}^{k}\right\}, & \text { if in Case 1, } \\
\left\{t^{\prime} \in \mathbb{F}_{q}^{k} \mid t_{k}=0\right\}, & \text { if in Case 2, first sum, } \\
\left\{t^{\prime} \in \mathbb{F}_{q}^{k} \mid t_{k} \in \mathbb{F}_{q}^{*}\right\}, & \text { if in Case 2, second sum, }\end{cases} \\
& f^{\tau} \text { becomes } \begin{cases}f^{(+0, \tau)}, & \text { if in Case 1, } \\
f^{(-0, \tau)}, & \text { if in Case 2, first sum, } \\
f^{(1, \tau)}, & \text { if in Case 2, second sum, }\end{cases} \\
& v^{\tau} \text { becomes } \begin{cases}v^{(+0, \tau)}, & \text { if in Case 1, first sum, } \\
v^{(-0, \tau)}, & \text { if in Case 2, fir } \\
v^{(1, \tau)}, & \text { if in Case 2, second sum. }\end{cases}
\end{aligned}
$$

Thus,

$$
\Xi^{\tau}(u, v)= \begin{cases}\Xi^{(+0, \tau)}(u, v), & \text { if Case 1, } \\ \Xi^{(-0, \tau)}(u, v)+\Xi^{(1, \tau)}(u, v), & \text { if Case 2, }\end{cases}
$$

as desired.

$$
\text { 5. The CASE } G=G L_{n}\left(\mathbb{F}_{q}\right)
$$

Let $G=G L_{n}\left(\mathbb{F}_{q}\right)$ be the general linear group over the finite field $\mathbb{F}_{q}$ with $q$ elements. This section uses braid-like diagrams to analyze multiplication in unipotent Hecke algebras. The structure of this section is as follows.

5.1 describes the braid-like diagrams of this paper, and how the Chevalley relations translate into diagram relations.

5.2 reviews unipotent Hecke algebras for $G L_{n}\left(\mathbb{F}_{q}\right)$ in this context, and shows how to identify the diagrams of unipotent Hecke algebra basis elements.

5.3 uses a 3 step process to multiply basis elements using the visual cues of the diagrams.

5.4 summarizes a complete algorithm for multiplying basis elements, and illustrates the process with a nontrivial example. 
Define subgroups

$$
\begin{aligned}
& T=\left\{\begin{array}{c}
\text { diagonal } \\
\text { matrices }
\end{array}\right\}, \quad N=\left\{\begin{array}{c}
\text { monomial } \\
\text { matrices }
\end{array}\right\},
\end{aligned}
$$

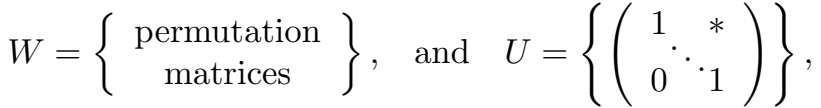

where a monomial matrix is a matrix with exactly one nonzero entry in each row and column.

Let $x_{i j}(t) \in U$ be the matrix with $t$ in position $(i, j)$, ones on the diagonal and zeroes elsewhere; write $x_{i}(t)=x_{i, i+1}(t)$. Let $h_{\varepsilon_{i}}(t) \in T$ denote the diagonal matrix with $t$ in the $i$ th slot and ones elsewhere, and let $s_{i} \in W \subseteq N$ be the identity matrix with the $i$ th and $(i+1)$ st columns interchanged. That is,

$$
\begin{gathered}
x_{i}(t)=I d_{i-1} \oplus\left(\begin{array}{ll}
1 & t \\
0 & 1
\end{array}\right) \oplus I d_{n-i-1}, \quad h_{\varepsilon_{i}}(t)=I d_{i-1} \oplus(t) \oplus I d_{n-i}, \\
s_{i}=I d_{i-1} \oplus\left(\begin{array}{ll}
0 & 1 \\
1 & 0
\end{array}\right) \oplus I d_{n-i-1},
\end{gathered}
$$

where $I d_{k}$ is the $k \times k$ identity matrix. Then

$$
\begin{gathered}
W=\left\langle s_{1}, s_{2}, \ldots, s_{n-1}\right\rangle, \quad T=\left\langle h_{\varepsilon_{i}}(t) \mid 1 \leq i \leq n, t \in \mathbb{F}_{q}^{*}\right\rangle, \quad N=W T, \\
U=\left\langle x_{i j}(t) \mid 1 \leq i<j \leq n, t \in \mathbb{F}_{q}\right\rangle, \quad G=\langle U, W, T\rangle .
\end{gathered}
$$

The Chevalley group relations for $G$ are (see also Section 4.1)

(U1)

$$
\begin{aligned}
& x_{i j}(a) x_{r s}(b)=x_{r s}(b) x_{i j}(a) x_{i s}\left(\delta_{j r} a b\right) x_{r j}\left(-\delta_{i s} a b\right), \quad(i, j) \neq(r, s), \\
& x_{i j}(a) x_{i j}(b)=x_{i j}(a+b) \text {, } \\
& s_{i}^{2}=1, \\
& s_{i} s_{i+1} s_{i}=s_{i+1} s_{i} s_{i+1} \quad \text { and } \quad s_{i} s_{j}=s_{j} s_{i}, \\
& s_{i} h_{\varepsilon_{j}}(a)=h_{\varepsilon_{s_{i}(j)}}(a) s_{i},
\end{aligned}
$$

(N1)

(N2)

(N4) $\quad h_{\varepsilon_{i}}(b) h_{\varepsilon_{i}}(a)=h_{\varepsilon_{i}}(a b)$,

(N5) $\quad h_{\varepsilon_{j}}(b) h_{\varepsilon_{i}}(a)=h_{\varepsilon_{i}}(a) h_{\varepsilon_{j}}(b)$,

(IUN1) $s_{r} x_{i j}(t)=x_{s_{r}(i) s_{r}(j)}(t) s_{r}$,

(UN2) $\quad x_{i j}(a) h_{\varepsilon_{r}}(t)=h_{\varepsilon_{r}}(t) x_{i j}\left(t^{-\delta_{r i}} t^{\delta_{r j}} a\right)$,

where $\delta_{i j}$ is the Kronecker delta.

5.1. A pictorial version of $G L_{n}\left(\mathbb{F}_{q}\right)$. For the results that follow, it will be useful to view elements of $\mathbb{C} G$ as braid-like diagrams instead of matrices. The basic idea is to depict an $n \times n$ permutation matrix $w$ as two rows of $n$ vertices each, with an edge (called a strand) from the $i$ th top vertex to the $j$ th bottom vertex if $w(i)=j$. For example,

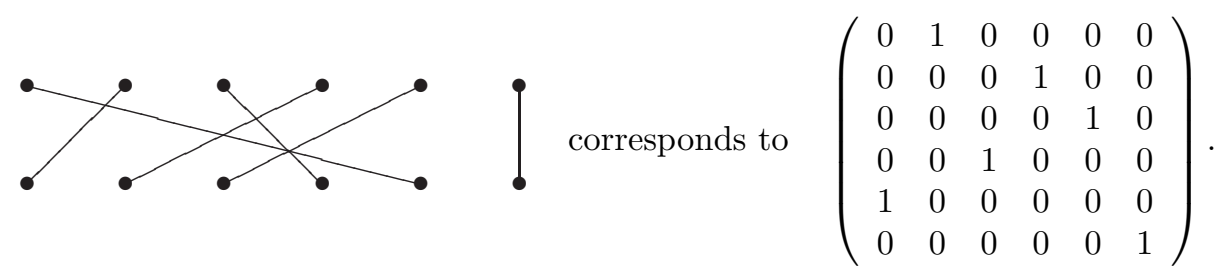


Matrix multiplication corresponds to concatenation of diagrams, so
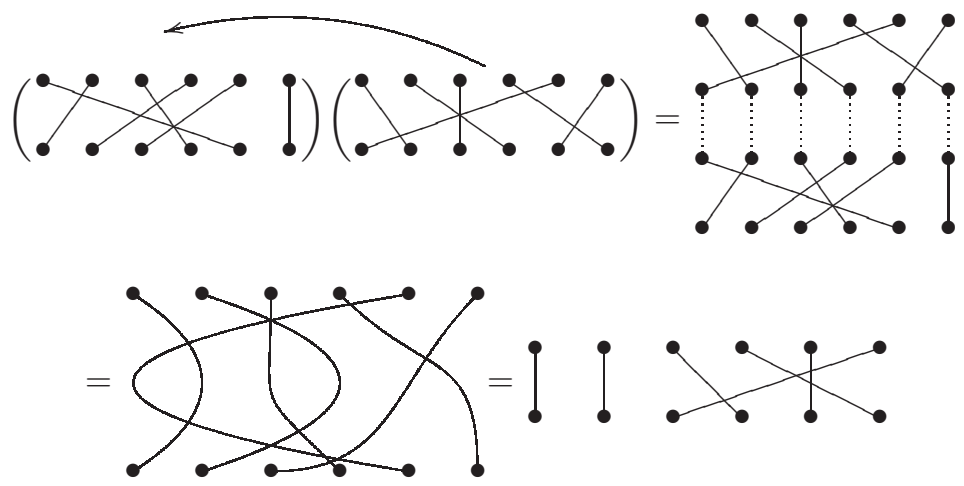

We generalize these diagrams to $G L_{n}\left(\mathbb{F}_{q}\right)$ by adding different varieties of "beads" to these diagrams that slide along the strands. A diagonal matrix corresponds to the identity permutation with a bead on each strand, such as

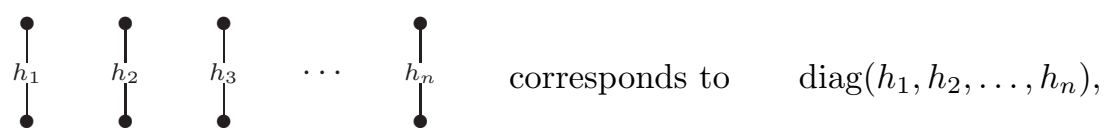

and we depict the matrix $x_{i j}(t)$ by the identity permutation with directed beads on the $i$ th and $j$ th strands, such as

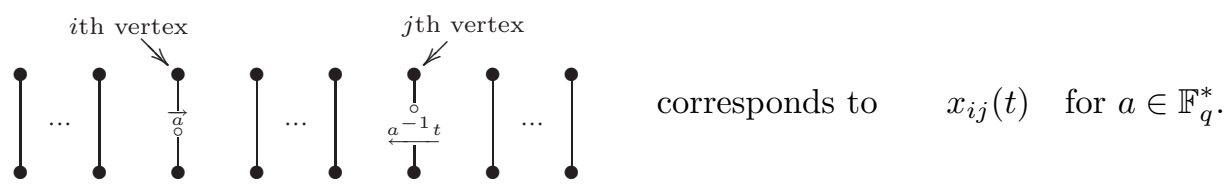

Note there is an implicit relation in this last correspondence given by

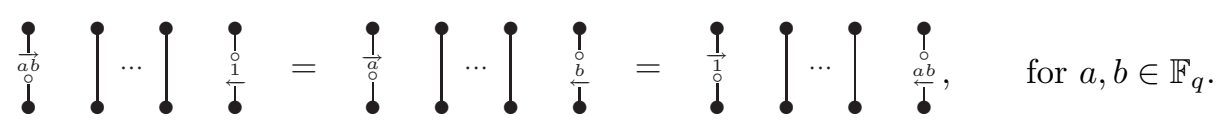

The advantage of this approach is that it allows a visual shortcut to computing products (such as the permutations above) and commutations in $G L_{n}\left(\mathbb{F}_{q}\right)$. For example, we can summarize multiple applications of (N3) by simply pushing the beads of $h \in T$ along the strands of $w \in W$ so that

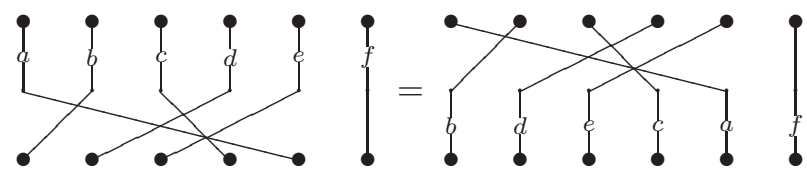

gives

$$
\begin{aligned}
& s_{4} s_{3} s_{4} s_{2} s_{3} s_{1} h_{\varepsilon_{1}}(a) h_{\varepsilon_{2}}(b) h_{\varepsilon_{3}}(c) h_{\varepsilon_{4}}(d) h_{\varepsilon_{5}}(e) h_{\varepsilon_{6}}(f) \\
& =h_{\varepsilon_{5}}(a) h_{\varepsilon_{1}}(b) h_{\varepsilon_{4}}(c) h_{\varepsilon_{2}}(d) h_{\varepsilon_{3}}(e) h_{\varepsilon_{6}}(f) s_{4} s_{3} s_{4} s_{2} s_{3} s_{1} .
\end{aligned}
$$


The generators of $G$ are

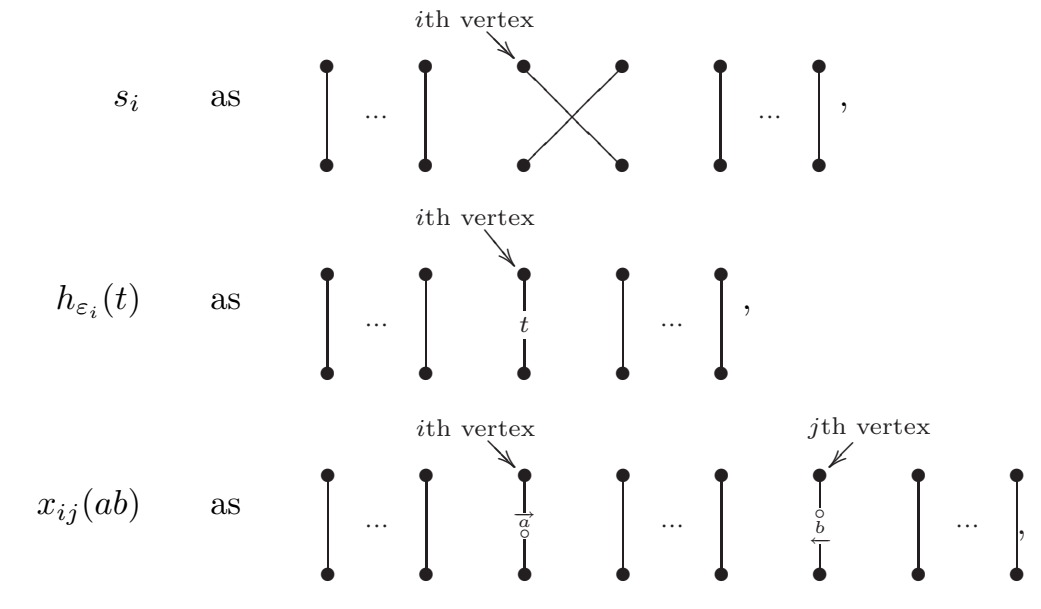

where each diagram has two rows of $n$ vertices. In the following Chevalley relations, curved strands indicate longer strands, so for example (UN1) indicates that $\vec{a}$ and $\stackrel{b}{b}$ slide along the strands they are on (no matter how long). The Chevalley relations translate to

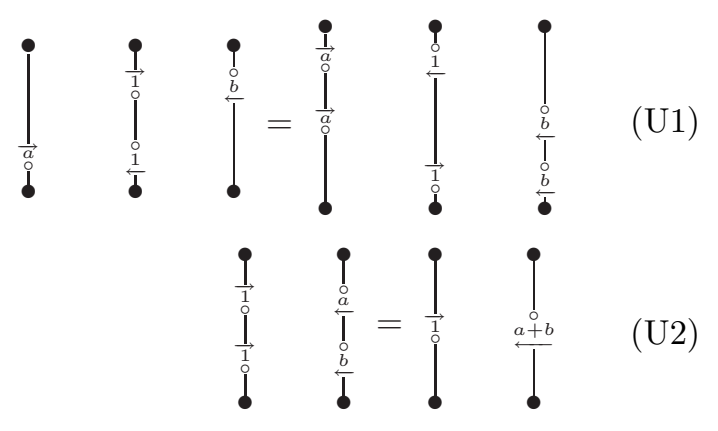

(the beads $\underset{a}{\vec{a}}$ and $\underset{b}{\vec{b}}$ commute unless two arrows or two circles encounter one-another on a strand).
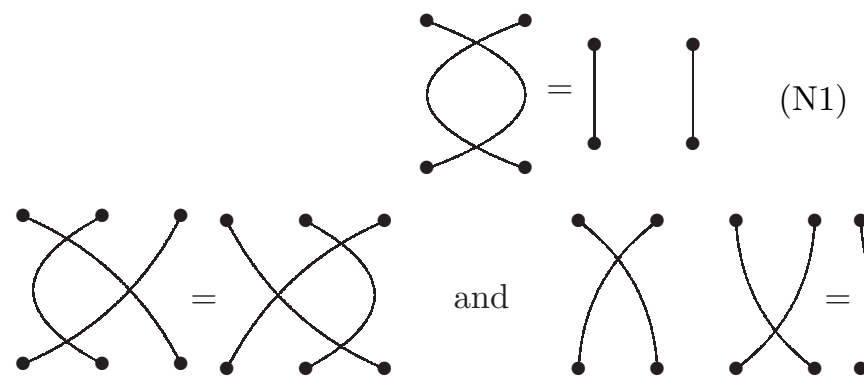

and

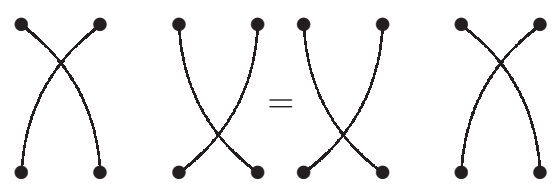


(relations in $W$ exactly describe what one can do by pushing the strands around the diagrams),

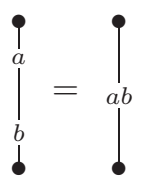

(N4)
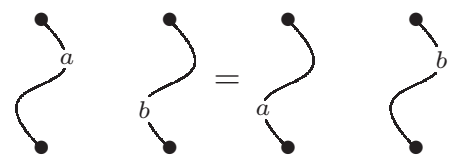

( $T$-type beads follow strands and multiply if they hit one another),
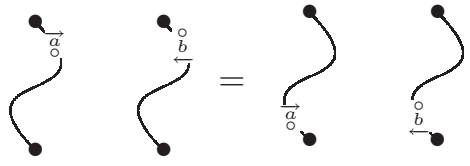

(UN1)
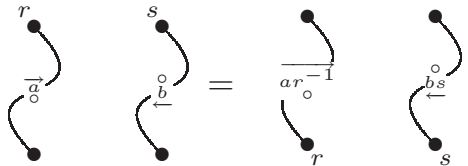

(UN2)

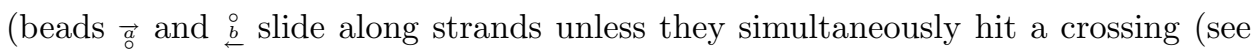
(UN3) below), and the circle or arrow determine how $T$-type beads interact with $\vec{a}$ and $\stackrel{\circ}{b})$,

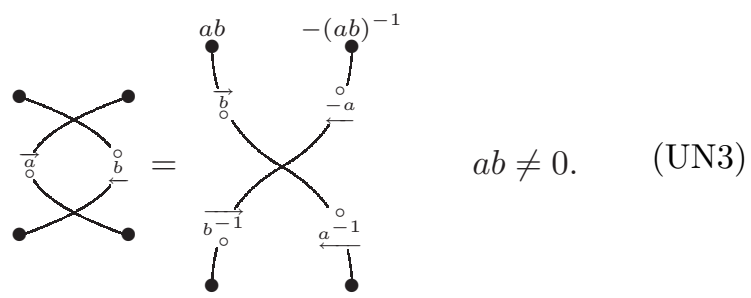

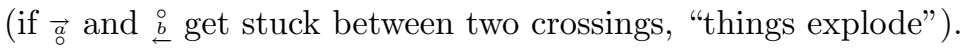

5.2. The unipotent Hecke algebra $\mathcal{H}_{\mu}$. Fix a nontrivial group homomorphism $\psi: \mathbb{F}_{q}^{+} \rightarrow \mathbb{C}^{*}$, fix a map

$$
\mu:\{1,2, \ldots, n-1\} \quad \longrightarrow \quad\{0,1\}
$$

and define

$$
\mu_{i j}= \begin{cases}\mu_{i}, & \text { if } j=i+1 \\ 0, & \text { otherwise }\end{cases}
$$

Then

$$
\begin{aligned}
& \psi_{\mu}: \quad U \quad \longrightarrow \quad \mathbb{C}^{*} \\
& x_{i j}(t) \mapsto \psi\left(\mu_{i j} t\right)
\end{aligned}
$$

is a group homomorphism.

The unipotent Hecke algebra $\mathcal{H}_{\mu}$ of the triple $\left(G, U, \psi_{\mu}\right)$ is

$$
\mathcal{H}_{\mu}=\operatorname{End}_{G}\left(\operatorname{Ind}_{U}^{G}\left(\psi_{\mu}\right)\right) \cong e_{\mu} \mathbb{C} G e_{\mu}, \quad \text { where } \quad e_{\mu}=\frac{1}{|U|} \sum_{u \in U} \psi_{\mu}\left(u^{-1}\right) u .
$$

If $N_{\mu}=\left\{v \in N \mid e_{\mu} v e_{\mu} \neq 0\right\}$, then $\left\{e_{\mu} v e_{\mu} \mid v \in N_{\mu}\right\}$ is a basis for $\mathcal{H}_{\mu}$. 
We may characterize the elements of $N_{\mu}$ in the following fashion (for a more extensive analysis of $N_{\mu}$ see [Th]). Suppose $v \in N$. For each $\mu_{i}=0$, place a dotted line between the $i$ th and $(i+1)$ st vertices; for example, $\mu=(1,0,1,1,0,0)$ gives:

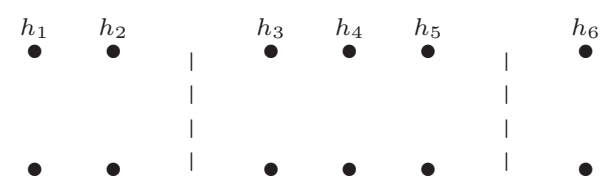

Then $e_{\mu} v e_{\mu} \neq 0$ if and only if the diagram for $v$ satisfies

if

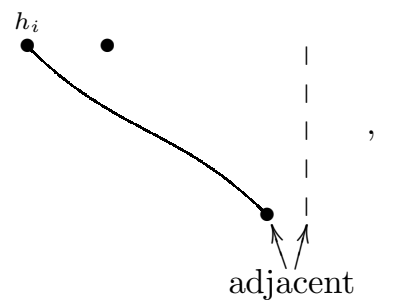

adjacent

then

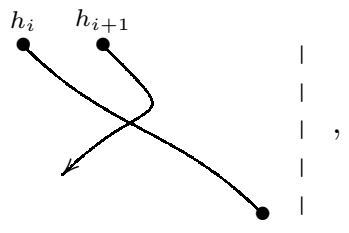

(2)

if
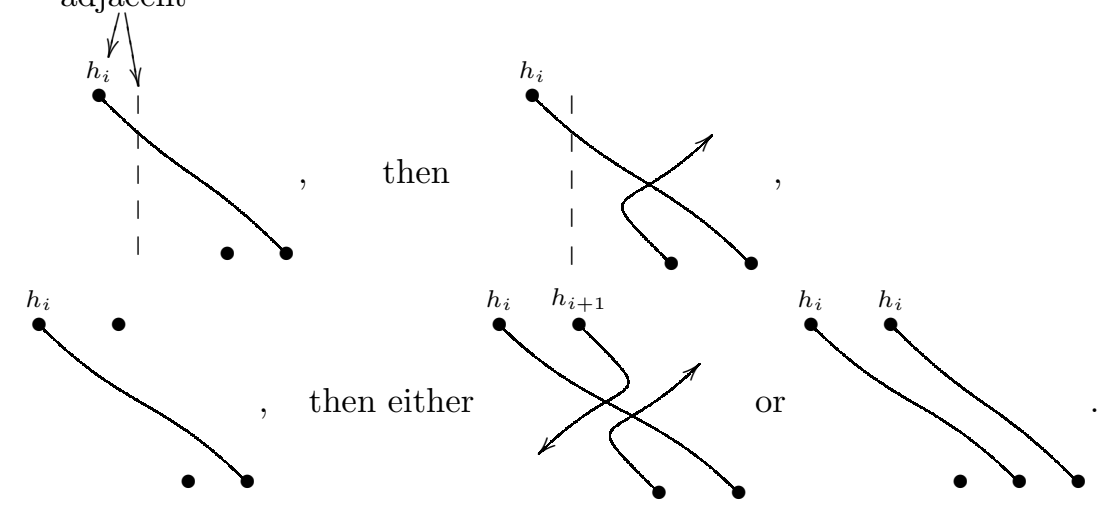

Example. If $\mu=(1,0,1,1,1,1,0,1,1,0,1,1,1,0)$ then

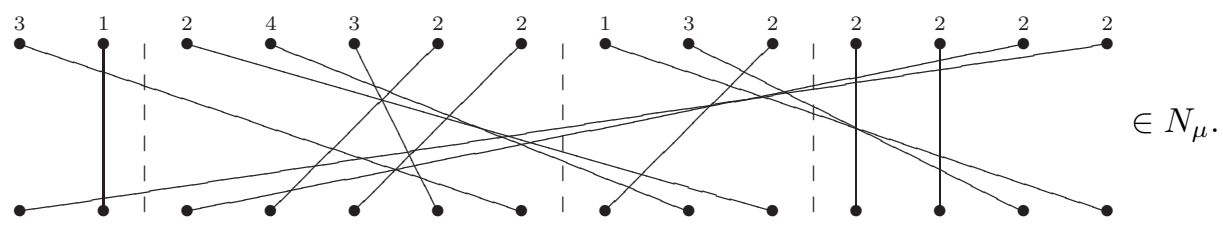

Note that the map

$$
\begin{aligned}
\pi: N=W T & \longrightarrow W \\
w h & \mapsto w, \quad \text { for } w \in W, h \in T,
\end{aligned}
$$

is a surjective group homomorphism. Let $u \in N$ with $\pi(u)=s_{i_{1}} \cdots s_{i_{r}}$ for $r$ minimal. Then there is a unique $u_{T} \in T$ such that

$$
u=u_{1} u_{2} \cdots u_{r} u_{T}, \quad \text { where } u_{k}=s_{i_{k}} .
$$

We write $u_{k}$ instead of $s_{i_{k}}$ because when working with diagrams, it is clear where the crossing is located and it is more important to determine the order in which 
order the crossings come, as in

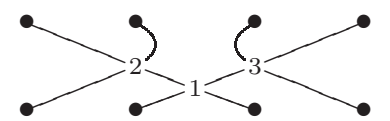

or

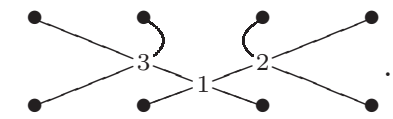

For $t \in \mathbb{F}_{q}$, write $u_{k}(t)=s_{i_{k}} x_{i_{k}}(t)$.

For any $\mu$ as in (5.7), the decomposition

$$
U=\prod_{1 \leq i<j \leq n} U_{i j}, \quad \text { where } \quad U_{i j}=\left\langle x_{i j}(t) \mid t \in \mathbb{F}_{q}\right\rangle
$$

implies

$$
e_{\mu}=\prod_{1 \leq i<j \leq n} e_{i j}\left(\mu_{i j}\right), \quad \text { where } \quad e_{i j}(k)=\frac{1}{q} \sum_{t \in \mathbb{F}_{q}} \psi(-k t) x_{i j}(t)
$$

Pictorially,

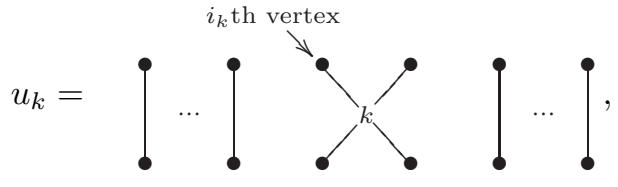

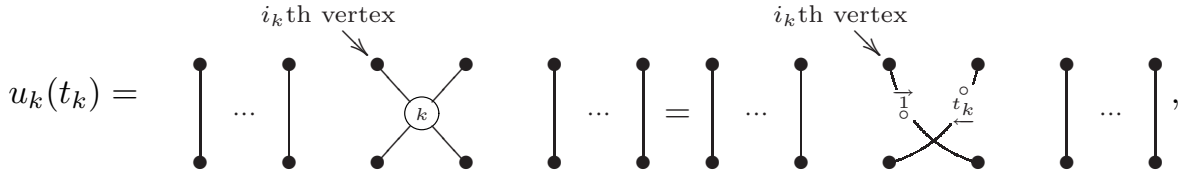

$$
\begin{aligned}
& e_{i j}(k)=\overbrace{\cdots}^{i \text { th vertex }}
\end{aligned}
$$

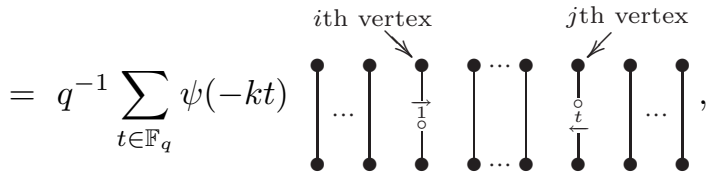

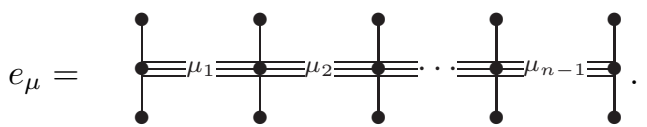

Therefore, if $n=5$, since

$$
e_{\mu}=e_{13}(0) e_{23}\left(\mu_{2}\right) e_{12}\left(\mu_{1}\right) e_{45}\left(\mu_{4}\right) e_{15}(0) e_{25}(0) e_{14}(0) e_{35}(0) e_{24}(0) e_{34}\left(\mu_{3}\right)
$$


it follows that

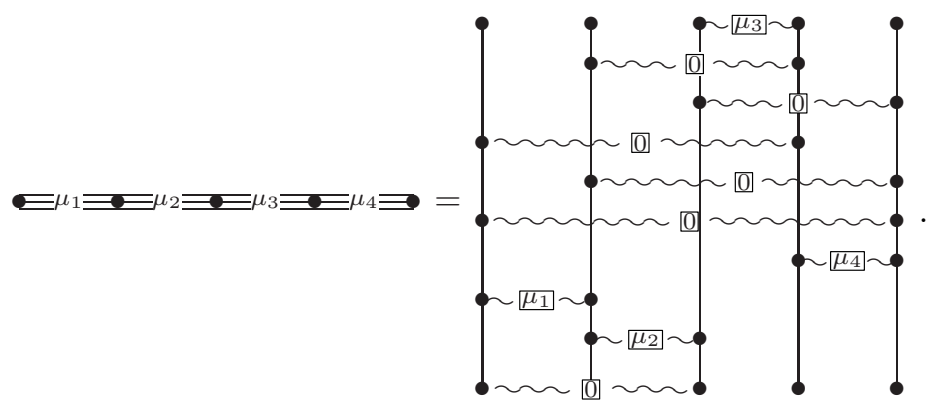

A running example. Throughout this section we will illustrate points using the example

$$
u=u_{1} u_{2} u_{3} u_{4} u_{5} u_{6} u_{7} u_{8} u_{T} \in N_{5} \quad \text { according to } s_{3} s_{1} s_{2} s_{3} s_{1} s_{4} s_{2} s_{3} \in S_{5},
$$

with $u_{T}=\operatorname{diag}(a, b, c, d, e)$. In this case,

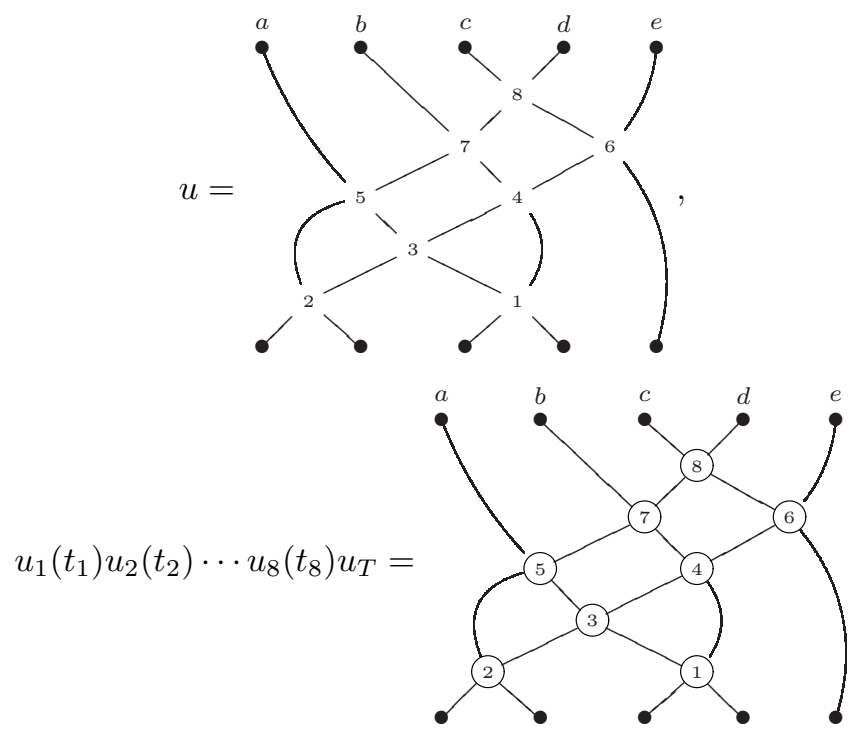

and

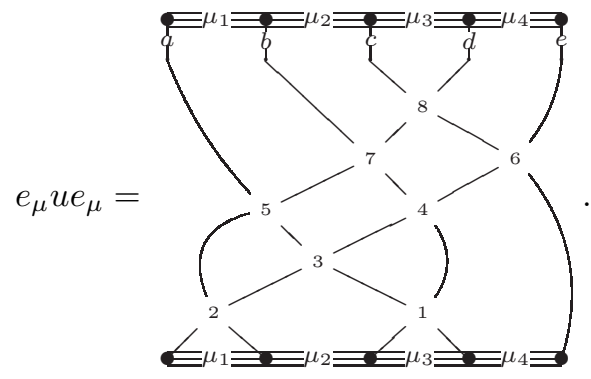


The elements $e_{i j}(k)$ also interact with $U$ and $N$ as follows (see also Section 4.1):

(E1)

$$
\begin{aligned}
& s_{r} e_{i j}(k) s_{r}=e_{s_{r}(i) s_{r}(j)}(k), \\
& \text { (E2) } \quad e_{\mu} v e_{i j}\left(\mu_{i j}\right)=e_{\mu} v, \quad v \in N_{\mu},(\pi v)(i)<(\pi v)(j) \text {, } \\
& \text { (E3) } e_{i j}(k) h_{\varepsilon_{l}}(r)=h_{\varepsilon_{l}}(r) e_{i j}\left(k r^{\delta_{l i}} r^{-\delta_{l j}}\right) \text {, } \\
& \text { (E4) } \quad e_{\mu} x_{i j}(t)=\psi\left(\mu_{i j} t\right) e_{\mu}=x_{i j}(t) e_{\mu} \text {, }
\end{aligned}
$$

or pictorially,

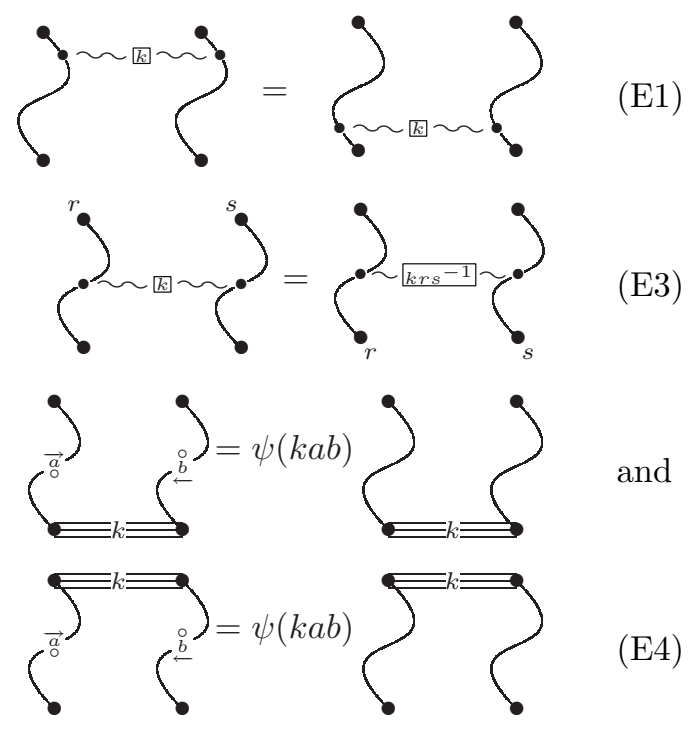

and for $v \in N_{\mu}$ with $(\pi v)(i)<(\pi v)(j)$, and for $v \in N_{\mu}$ with $(\pi v)(i)<(\pi v)(j)$,
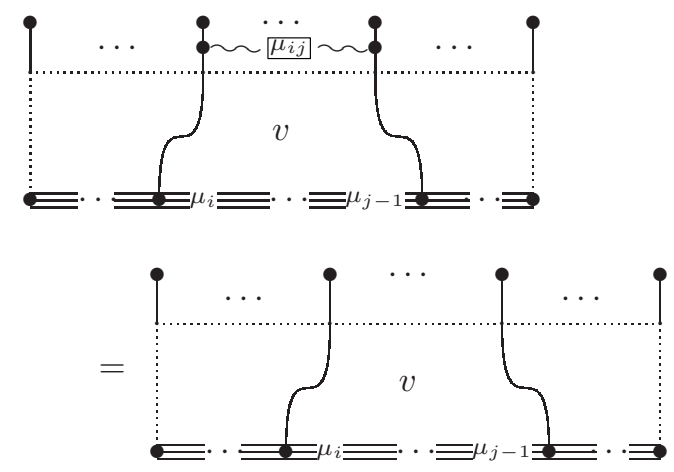

5.3. Basis element multiplication using braids. When we multiply two basis elements $e_{\mu} u e_{\mu}$ and $e_{\mu} v e_{\mu}$, the product $e_{\mu} u e_{\mu} v e_{\mu}$ has an $e_{\mu}$ "stuck" between the $u$ 
and the $v$, or:

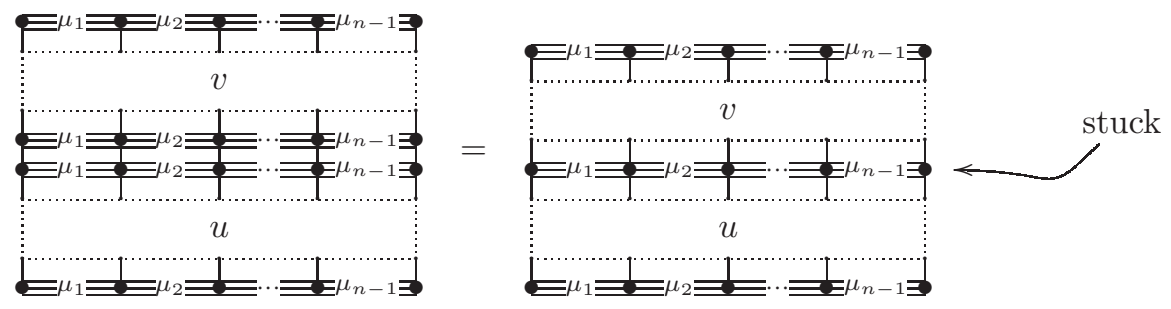

We then use the Chevalley relations to piece by piece "push" the center $e_{\mu}$ to the outside of the diagram. The first step is to push the $e_{\mu}$ as far into $u$ as possible, as illustrated by the following example.

Example (see (5.18) ). Let $u=u_{1} u_{2} \cdots u_{8} u_{T} \in N$ according to $s_{3} s_{1} s_{2} s_{3} s_{1} s_{4} s_{2} s_{3} \in$ $W$ and $u_{T}=\operatorname{diag}(a, b, c, d, e) \in T$. By (5.17), we may write

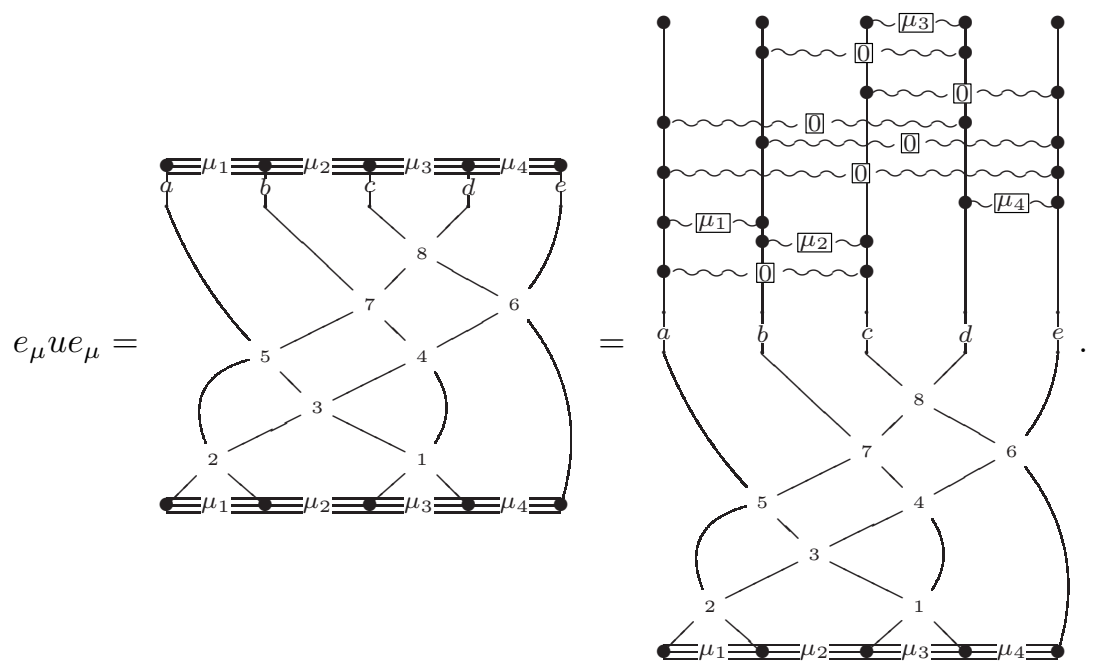

Note that the strands that $e_{13}(0)$ and $e_{23}\left(\mu_{2}\right)$ connect never cross, so we can use (E2) to push them through the diagram of $u$. The rest of the $e_{i j}(k)$ get stuck on 
some crossing, so we use (E3) to first move $u_{T}$ through the remaining $e_{i j}(k)$ :

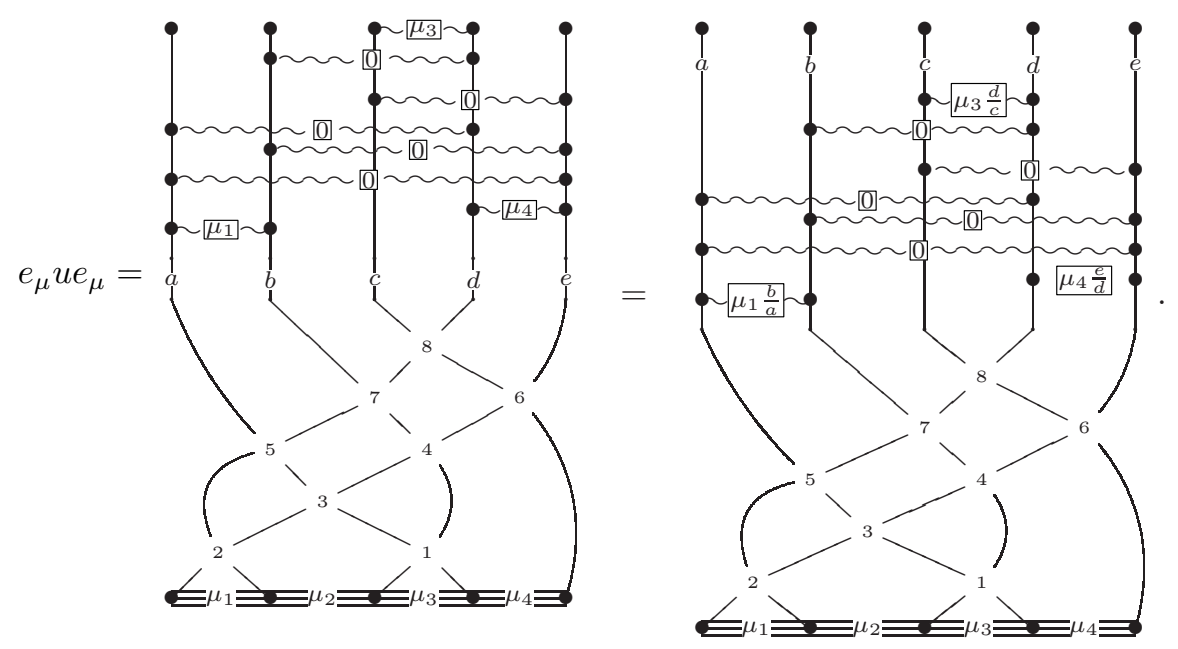

Next, use (E1) to push the $e_{i j}(k)$ down into $u$ until the strands they are on cross,
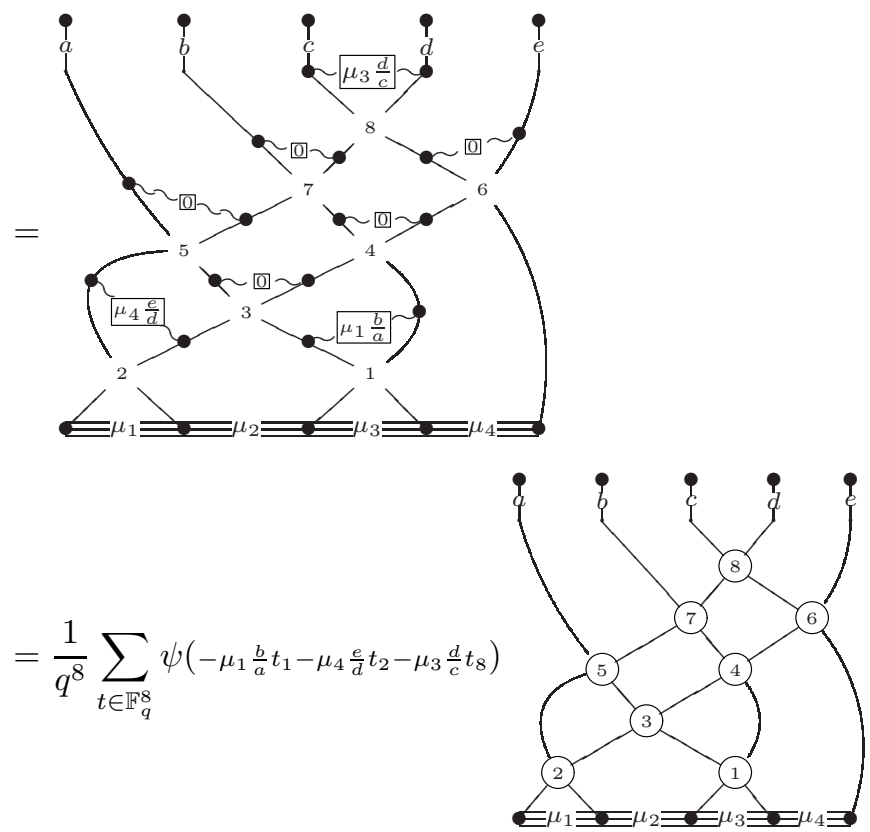

by definitions (5.15), (5.13), and (5.14).

Step 0: Push $e_{\mu}$ into the diagram $u$. Suppose $u=u_{1} u_{2} \cdots u_{r} u_{T} \in N_{\mu}$ with $u_{T}=\operatorname{diag}\left(h_{1}, h_{2}, \ldots, h_{n}\right)$. As illustrated in the example above, use (5.12), (E3), 
(E1) and (E2) to rewrite $e_{\mu} u e_{\mu}$ as
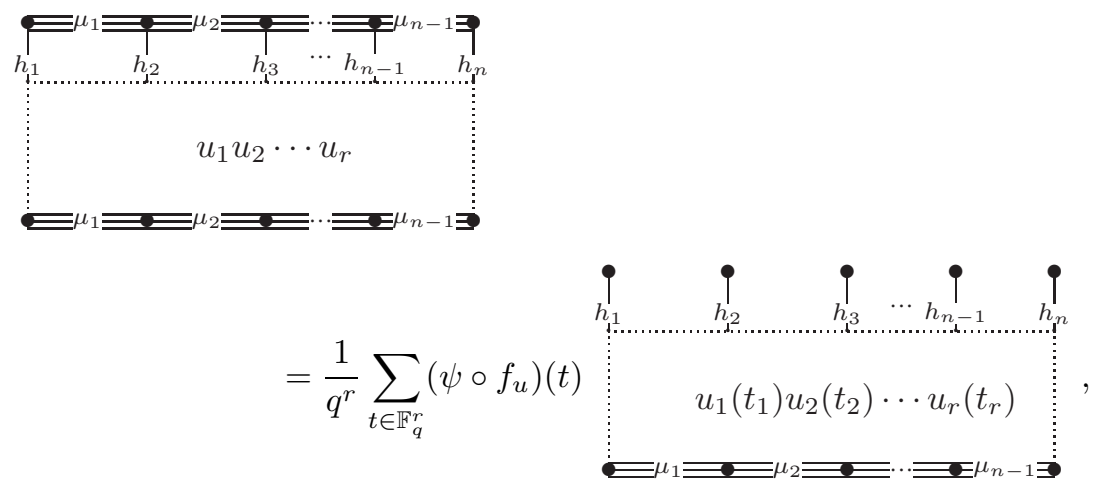

where $f_{u} \in \mathbb{F}_{q}\left[y_{1}, y_{2}, \ldots, y_{r}\right]$ is given by

$$
f_{u}\left(y_{1}, y_{2}, \ldots, y_{r}\right)=-\mu_{i_{1} j_{1}} h_{i_{1}}^{-1} h_{j_{1}} y_{1}-\mu_{i_{2} j_{2}} h_{i_{2}}^{-1} h_{j_{2}} y_{2}-\cdots-\mu_{i_{r} j_{r}} h_{i_{r}}^{-1} h_{j_{r}} y_{r}
$$

where $\left(i_{k}, j_{k}\right)=(a, b)$, if the $k$ th crossing in $u$ crosses the strands coming from the $a$ th and $b$ th top vertices.

Note that relation (5.20) can be quickly computed by visually ascertaining which strands cross in the diagram.

Step 1: Concatenate $\left(e_{\mu} u e_{\mu}\right)$ with $\left(v e_{\mu}\right)$. Let $u=u_{1} u_{2} \cdots u_{r} u_{T} \in N_{\mu}$ according to a minimal expression in $W$ as in (5.11). Let $v \in N_{\mu}$ and use (N3) and (N4) to write $u_{T} v=w \cdot \operatorname{diag}\left(a_{1}, a_{2}, \ldots, a_{n}\right)$, where $w=\pi(v) \in W$ (see (5.7)). Then use (5.20) to write

$$
\begin{aligned}
& \left(e_{\mu} u e_{\mu}\right)\left(e_{\mu} v e_{\mu}\right)=\left(e_{\mu} u e_{\mu}\right)\left(v e_{\mu}\right)
\end{aligned}
$$

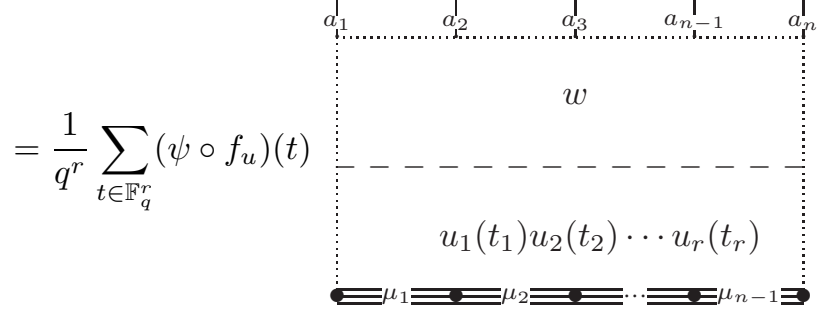

(This form corresponds to $\Xi^{\emptyset}\left(u, u_{T} v\right)$ of Corollary [3) 
Example (continued). If $u$ is as in (5.18) and

$$
v=s_{2} s_{3} s_{2} s_{1} s_{2} \cdot \operatorname{diag}(f, g, h, i, j) \in N,
$$

then by (5.19) and (제),

$$
\left(e_{\mu} u e_{\mu}\right)\left(e_{\mu} v e_{\mu}\right)=\left(e_{\mu} u e_{\mu}\right)\left(v e_{\mu}\right)
$$

is equal to

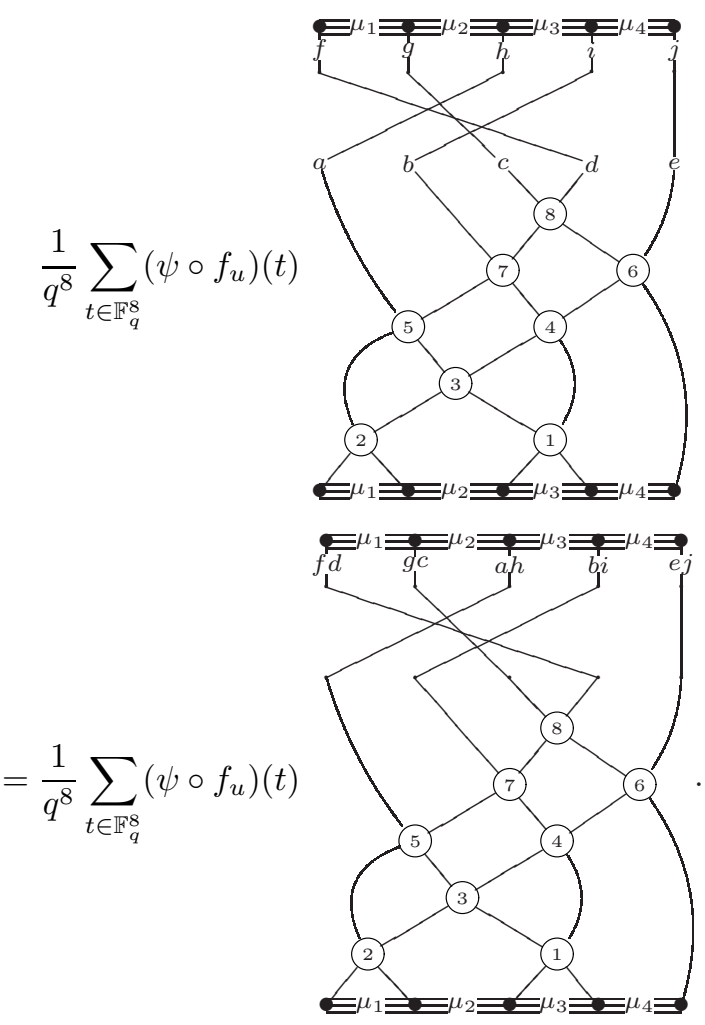

Step 2: Apply "braid" relations. Consider the crossing in (5.22) corresponding to $u_{r}\left(t_{r}\right)$ (the top crossing of $u$ ). There are two possibilities.

Case 1. the strands that cross at $(P$ do not cross again as they go up to the top of the diagram $\left(\ell\left(u_{r} w\right)>\ell(w)\right)$,

Case 2. the strands that cross at $(P)$ cross once on the way up to the top of the diagram $\left(\ell\left(u_{r} w\right)<\ell(w)\right)$. 
Relation 1 (Case 1). By (UN1), (UN2) and (E4), $\left(e_{\mu} u e_{\mu}\right)\left(v e_{\mu}\right)$ is equal to

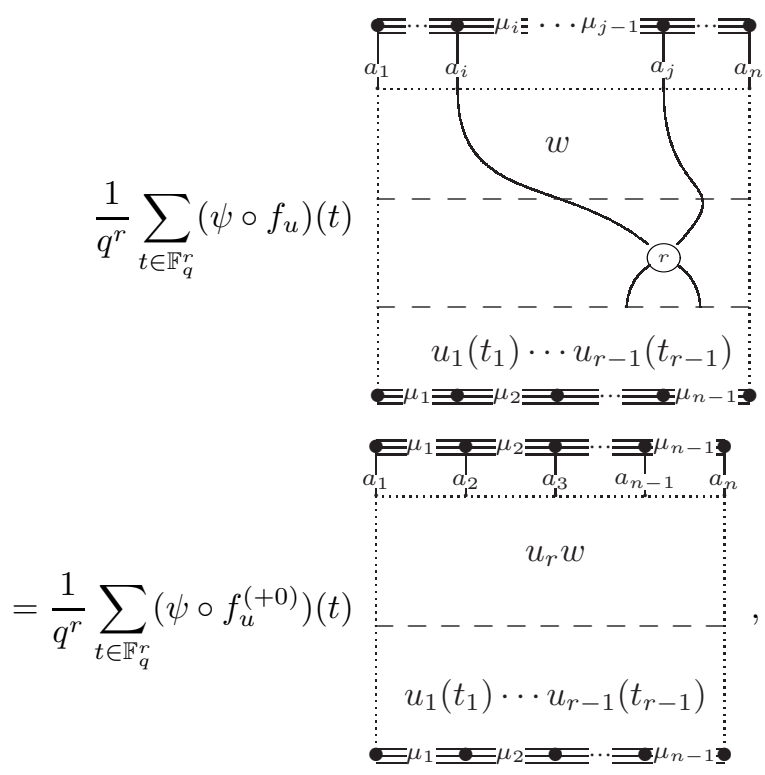

where $f_{u}^{(+0)}=f_{u}+\mu_{i j} a_{i}^{-1} a_{j} y_{r}$. Note that $f_{u}^{(+0)}=f_{u}$ unless $j=i+1$.

Relation 2 (Case 2). In Case 2,

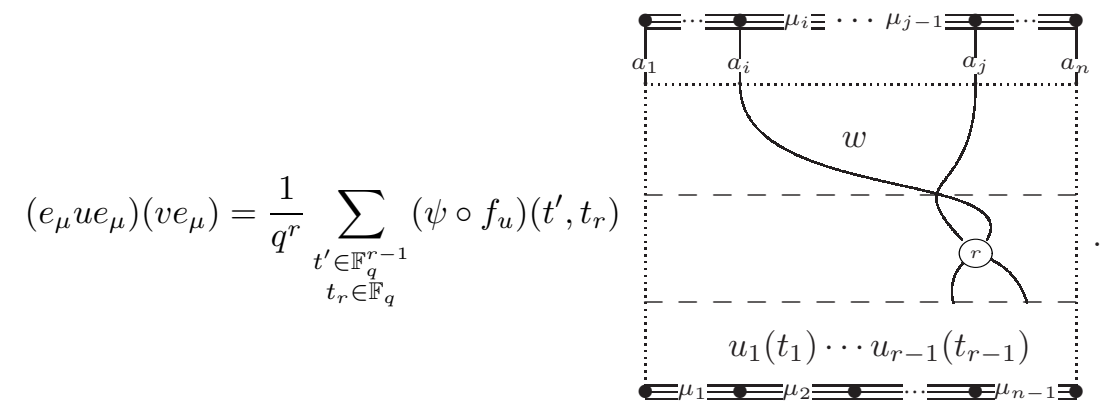

Split the sum into two parts corresponding to $t_{r}=0$ and $t_{r} \neq 0$ to get

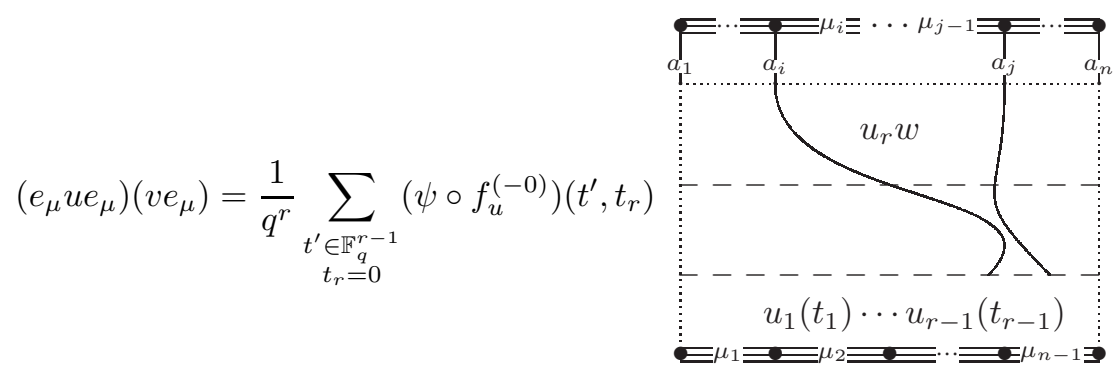


(by (N1))

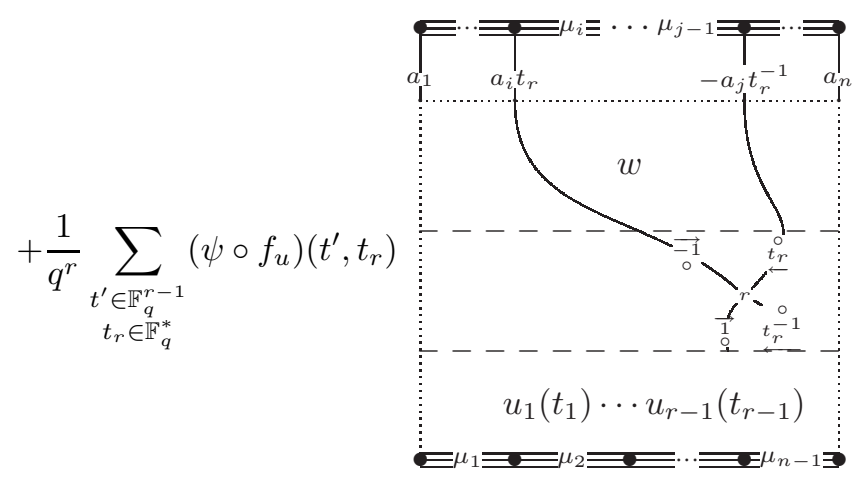

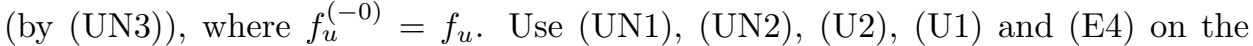

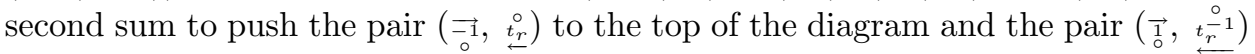
to the bottom.

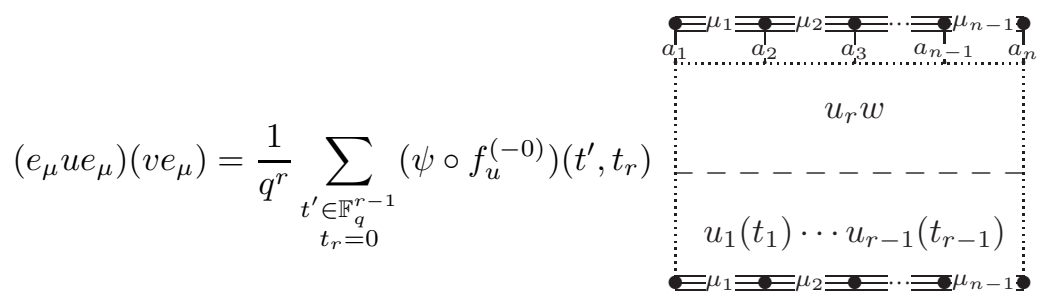

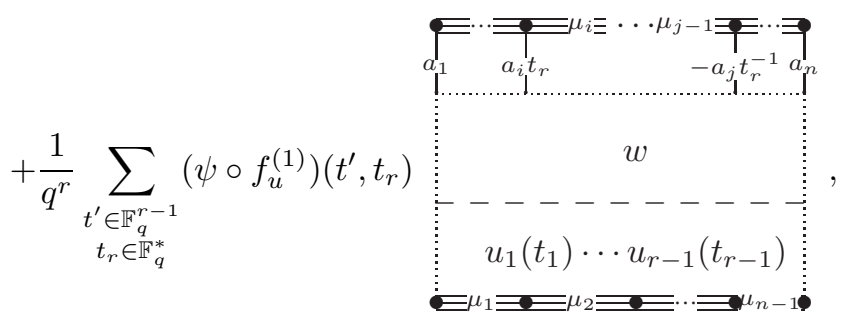

where $f_{u}^{(1)}=\varphi_{r}\left(f_{u}\right)+\mu_{i j} a_{j} a_{i}^{-1} y_{r}^{-1}$, and $\varphi_{r}(f)$ is defined by

(*) $\sum_{\substack{t \in \mathbb{F}_{q}^{r} \\ t_{r} \in \mathbb{F}_{q}^{*}}}(\psi \circ f)(t)$

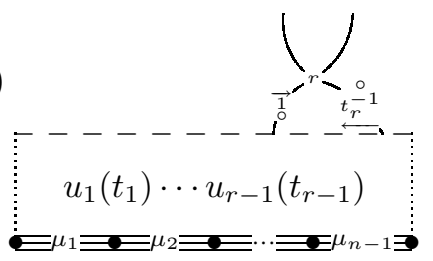

$$
=\sum_{\substack{t \in \mathbb{F}_{q}^{r} \\ t_{r} \in \mathbb{F}_{q}^{*}}}\left(\psi \circ \varphi_{r}(f)\right)(t)
$$

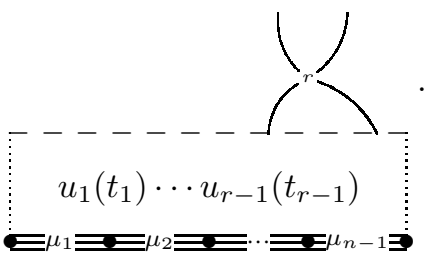


Remarks.

(a) We could have applied these steps for any $f, u$, and $v$, so we can iterate the process with each sum.

(b) The most complex step in these computations is determining $\varphi_{r}$. The following section develops a combinatorial method for computing the righthand side of (図).

Step $2^{\prime}:$ A combinatorial way to compute $\varphi_{k}$. Relation (函) pushes the beads $\overrightarrow{\stackrel{1}{\circ}}$ and $t_{r}^{\frac{0}{r}}$ through the diagram until they get to the bottom. Along the way, the beads hit crossings and we either apply relation (U1), which leads to additional beads, or (U2), which forces us to renormalize. In the following, red paint corresponds to the strands traversed by beads of the form $\vec{a}$ and blue paint corresponds to strands

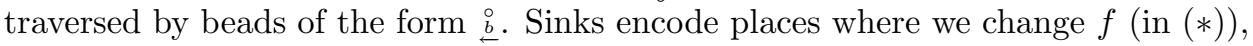
while paths and their weights describe how to change $f$. Lemma 5.1 below gives the resulting evaluation of the map $\varphi_{r}$ in (囝) .

Paint the strands below $u_{k}\left(u^{\circledR}\right)$. Suppose $u=u_{1} u_{2} \cdots u_{k} \in N$ decomposes according to $s_{i_{1}} s_{i_{2}} \cdots s_{i_{k}} \in W$ (assume $u_{T}=1$ ). Each step is illustrated with example (5.18).

(1) Paint the left [respectively right] strand exiting (k) below red [blue] all the way to the bottom of the diagram.

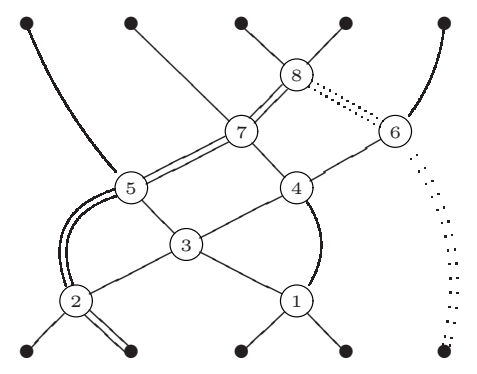

where red is $=$, blue is $\ldots \cdots \cdots \cdots$, , and $(k)$ is 8 .

(2) For each crossing that the red [blue] strand passes through, paint the right [left] strand (if possible) red [blue] until that strand either reaches the bottom or crosses the blue [red] strand of (1).

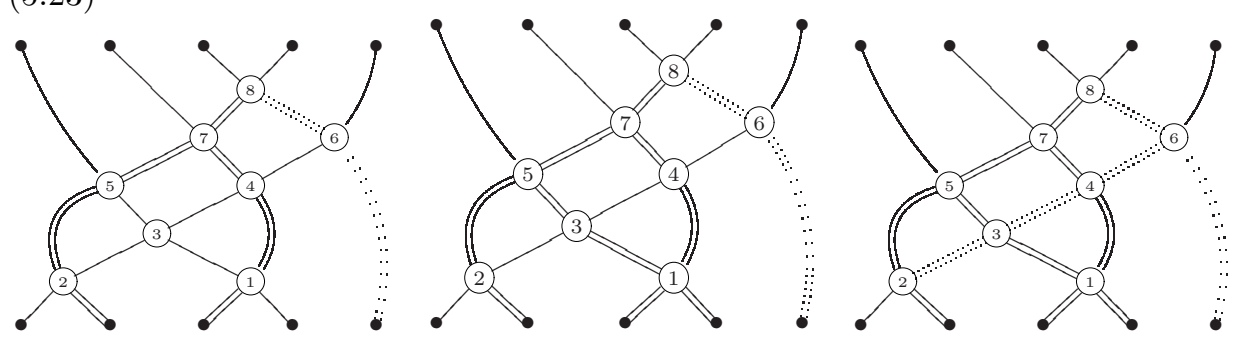

(3) Set

$u^{\circledR}=$ the diagram $u_{1}\left(t_{1}\right) u_{2}\left(t_{2}\right) \cdots u_{k}\left(t_{k}\right)$ painted according to (1) and (2). 
Sinks. The diagram $u^{(囚)}$ has a crossed sink at (j) if (j) is a crossing between a red strand and a blue one, or:

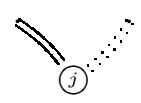

Note that since $u$ is decomposed according to a minimal expression in $W$, there will be no crossings of the form

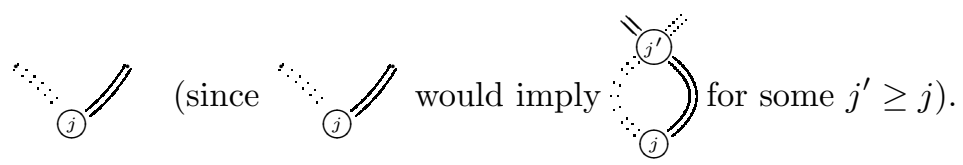

The diagram $u^{\left({ }^{\circledR}\right.}$ has a bottom sink at $j$ if a red strand enters the $j$ th bottom vertex and a blue strand enters the $(j+1)$ st bottom vertex, or:

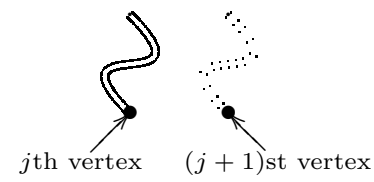

Example (continued). In the running example above $u^{(8)}$ has crossed sinks at (2), (3), and (4), and a bottom sink at 4 . Note that (1) is not a crossed sink since both strands are red.

Paths. A red [respectively blue] path $p$ from a sink $s$ (either crossed or bottom) in $u^{\circledR}$ is an increasing sequence

$$
j_{1}<j_{2}<\cdots<j_{l}=k
$$

such that in $u^{\circledR}$

(a) im is directly connected (no intervening crossings) to by a red [blue] strand,

(b) if $s$ is a crossed sink, then (11) $=s$,

$\left(\mathrm{b}^{\prime}\right)$ if $s$ is a bottom sink, then

- in a red path, the $s$ th bottom vertex connects to the crossing (11) with a red strand,

- in a blue path, the $(s+1)$ st bottom vertex connects to the crossing (11) with a blue strand. 
Example (continued). The sinks with their corresponding paths for $u^{(}$are

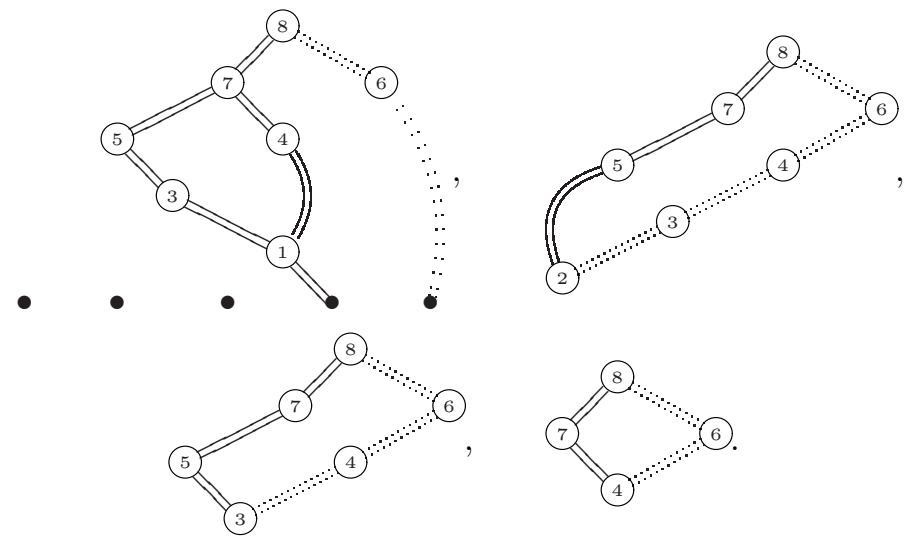

Let

$$
P=\left(u^{\circledR}, s\right)=\left\{\begin{array}{c}
\text { red paths from } \\
s \text { in } u^{\circledR}
\end{array}\right\} \quad \text { and } \quad P:\left(u^{\circledR}, s\right)=\left\{\begin{array}{c}
\text { blue paths from } \\
s \text { in } u^{\circledR}
\end{array}\right\} .
$$

The weight of a path $p$ is

$$
\operatorname{wt}(p)= \begin{cases}\prod_{\substack{p \text { switches } \\ \text { strands at (i) }}} y_{i}, \quad \text { if } p \in P_{=}\left(u^{\circledR}, s\right), \\ \prod_{\substack{p \text { switches } \\ \text { strands at (i) }}}\left(-y_{i}\right), \quad \text { if } p \in P_{:}\left(u^{\circledR}, s\right) .\end{cases}
$$

Each sink $s$ in $u^{\circledR}$ (either crossed (j) or bottom $j$ ) has an associated polynomial $g_{s} \in \mathbb{F}_{q}\left[y_{1}, y_{2}, \ldots, y_{k-1}, y_{k}^{-1}\right]$ given by

$$
g_{s}=\sum_{\substack{p \in P_{=}\left(u^{\circledR}, s\right) \\ p^{\prime} \in P_{:}\left(u^{\circledR}, s\right)}} \operatorname{wt}(p) y_{k}^{-1} \mathrm{wt}\left(p^{\prime}\right) .
$$

Example (continued). Consider the weights of the above paths:

\begin{tabular}{|c|c|c|c|c|c|}
\hline Sink & 4 & 4 & 4 & (2) & (2) \\
\hline Path & $1<3<5<7<8$ & $1<4<7<8$ & $6<8$ & $2<5<7<8$ & $2<3<4<6<8$ \\
\hline Weight & $y_{5}$ & $y_{1} y_{7}$ & 1 & 1 & $-y_{6}$ \\
\hline \hline Sink & $(3$ & 0 & (4) & (4) & \multicolumn{1}{|}{} \\
\hline Path & $3<5<7<8$ & $3<4<6<8$ & $4<7<8$ & $4<6<8$ \\
\hline Weight & $y_{5}$ & $-y_{6}$ & $y_{7}$ & $-y_{6}$ \\
\hline
\end{tabular}

The corresponding polynomials are (5.28)

$$
g_{4}=y_{5} y_{8}^{-1}+y_{1} y_{7} y_{8}^{-1}, \quad g_{(2)}=-y_{8}^{-1} y_{6}, \quad g_{3}=-y_{5} y_{8}^{-1} y_{6}, \quad g_{(4)}=-y_{7} y_{8}^{-1} y_{6} .
$$

Lemma 5.1. Let $u=u_{1} u_{2} \cdots u_{r}$ and $\varphi_{r}$ be as in (R2) and (囷); suppose $u^{\odot}$ is painted as above. Then

$$
\varphi_{r}(f)=\left.f\right|_{\left\{y_{j} \mapsto y_{j}-g_{(7)} \mid(3) \text { a crossed sink }\right\}}+\sum_{j \text { a bottom }} \mu_{j} g_{j} .
$$


Proof. In the painting,

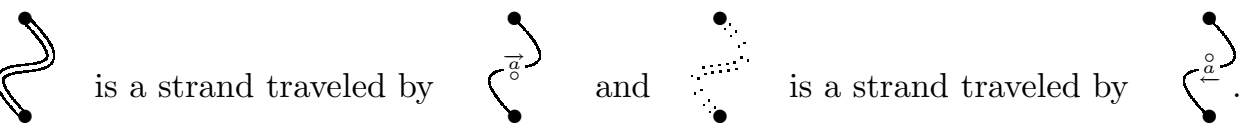

Substitutions due to crossed sinks correspond to the normalizations in relation (U2), and the sum over bottom sinks comes from applications of relation (E4).

For example (see (5.28)),

$$
\varphi_{8}(f)=\left.f\right|_{\substack{y_{4} \mapsto y_{4}-g_{(} \\ y_{3} \mapsto y_{3}-g_{3} \\ y_{2} \mapsto y_{2}-g_{(2)}}}+\mu_{4} g_{4}=\left.f\right|_{\substack{y_{4} \mapsto y_{4}+y_{7} y_{8}^{-1} y_{6} \\ y_{3} \mapsto y_{3}+y_{5} y_{8}^{-1} y_{6} \\ y_{2} \mapsto y_{2}+y_{8}^{-1} y_{6}}}+\mu_{4}\left(y_{5} y_{8}^{-1}+y_{1} y_{7} y_{8}^{-1}\right) .
$$

\subsection{A multiplication algorithm.}

Theorem 5.1 (The algorithm). Let $G=G L_{n}\left(\mathbb{F}_{q}\right)$ and $u, v \in N_{\mu}$. An algorithm for multiplying $e_{\mu} u e_{\mu}$ and $e_{\mu} v e_{\mu}$ is

(1) Decompose $u=u_{1} u_{2} \cdots u_{r} u_{T}$ according to some minimal expression in $W($ as in (5.11) $)$.

(2) Put $e_{\mu} u e_{\mu} v e_{\mu}$ into the form specified by (5.22), with $u_{T} v=w$. $\operatorname{diag}\left(a_{1}, a_{2}, \ldots, a_{n}\right)(w=\pi(v) \in W)$.

(3) Complete the following:

(a) If $\ell\left(u_{r} w\right)>\ell(w)$, then apply relation (R1).

(b) If $\ell\left(u_{r} w\right)<\ell(w)$, then apply relation ( $(\overline{R 2})$, using $\left(u_{1} u_{2} \cdots u_{r}\right)^{\oplus}$ and Lemma 5.1 to compute $\varphi_{r}$.

(4) If $r>1$, then reapply (3) to each sum with $r:=r-1$ and with

(a) $w:=u_{r} w$, after using (3a) or using (3b), in the first sum,

(b) $w:=w$, after using (3b), in the second sum.

(5) Set all diagrams not in $N_{\mu}$ to zero.

Sample computation. Suppose $n=3$ and $\mu_{i}=1$ for all $1 \leq i \leq 3$ (i.e. the Gelfand-Graev case). Then

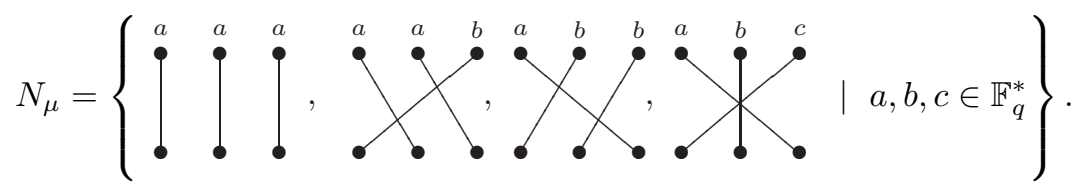

Suppose

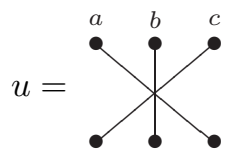

and

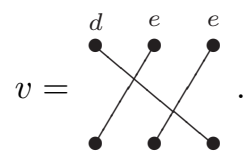

1. Theorem 5.11(1): Let $u=u_{1} u_{2} u_{3} u_{T} \in N_{\mu}$ decompose according to $s_{2} s_{1} s_{2} \in$ $W$, with $u_{T}=\operatorname{diag}(a, b, c)$. 
2. Theorem 5.1(2): By (5.22)
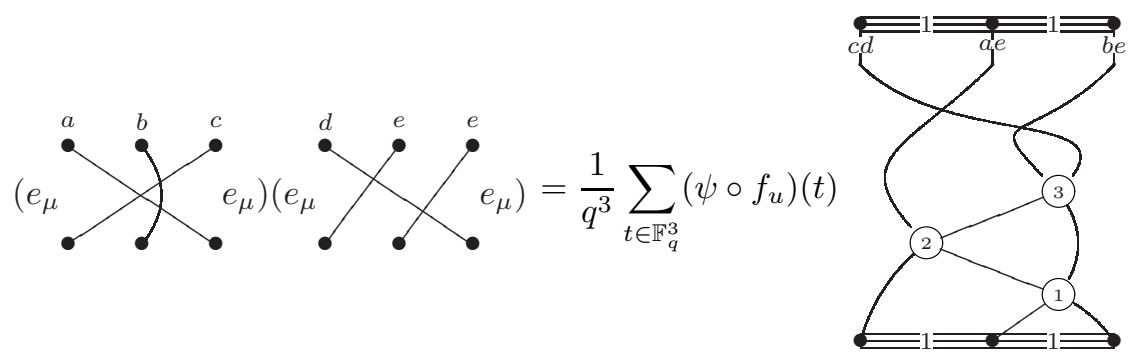

with $u_{T} v=s_{2} s_{1} \cdot \operatorname{diag}(c d, a e, b e)$ (so $\left.w=s_{2} s_{1}\right)$, and $f_{u}=-\frac{b}{a} y_{1}-\frac{c}{b} y_{3}$ (as in (15.21)).

3. Theorem $\mathbf{5 . 1}(\mathbf{3 b})$ : Since $\ell\left(u_{3} w\right)<\ell(w)$, paint $u_{1}\left(t_{1}\right) u_{2}\left(t_{2}\right) u_{3}\left(t_{3}\right)$ to get $\left(u_{1} u_{2} u_{3}\right)^{3}$ (as in (5.24) $)$,

$$
=\frac{1}{q^{3}} \sum_{t \in \mathbb{F}_{q}^{3}}\left(\psi \circ f_{u}\right)(t)
$$

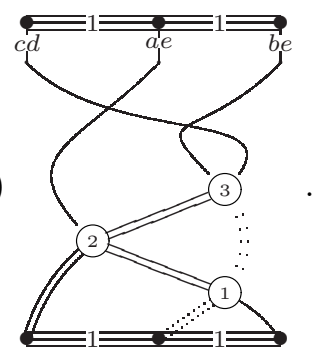

Now apply (R2),

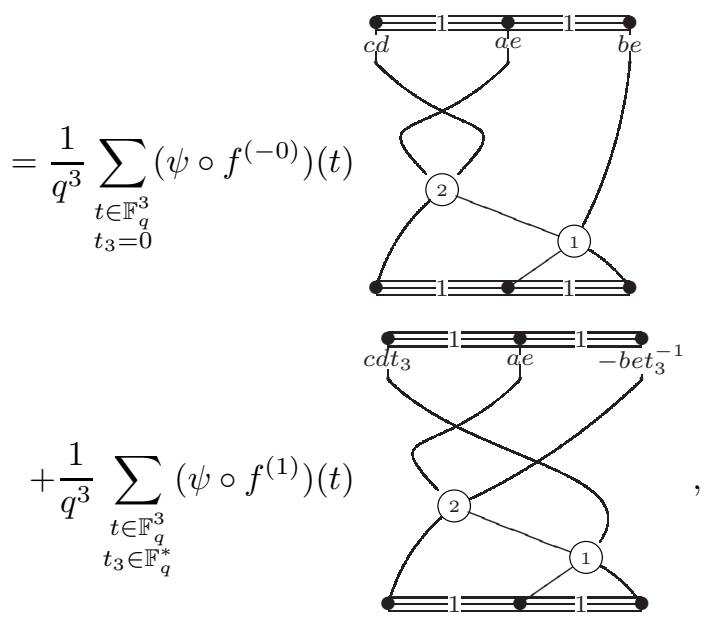

where $f^{(-0)}=-\frac{b}{a} y_{1}-\frac{c}{b} y_{3}$ and by Lemma 5.1 ,

$$
f^{(1)}=\varphi_{3}\left(f_{u}\right)+\mu_{13} \frac{b e}{c d} y_{3}^{-1}=-\frac{b}{a} y_{1}+\frac{b}{a} y_{2} y_{3}^{-1}-\frac{c}{b} y_{3}+y_{3}^{-1} .
$$

4. Theorem 5.1(4): Set $r:=2$ with $w:=u_{r} w=s_{1}$ in the first sum and $w:=w$ in the second sum.

5. Theorem [5.1(3a), (3b): In the first sum, $\ell\left(u_{2} s_{1}\right)<\ell\left(s_{1}\right)$, so paint $u_{1}\left(t_{1}\right) u_{2}\left(t_{2}\right)$ to get $\left(u_{1} u_{2}\right)^{2}$. In the second sum, $\ell\left(u_{2} s_{2} s_{1}\right)>\ell\left(s_{2} s_{1}\right)$, so apply 
(R1),

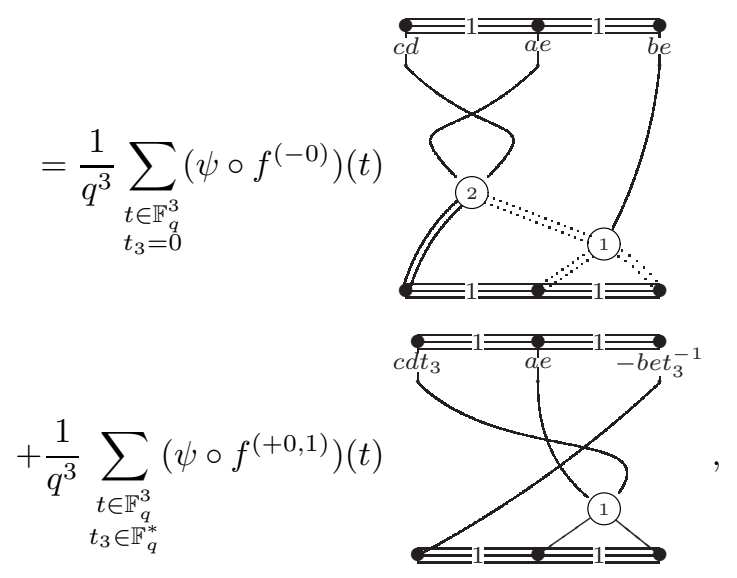

where $f^{(+0,1)}=-\frac{b}{a} y_{1}+\frac{b}{a} y_{2} y_{3}^{-1}-\frac{c}{b} y_{3}+y_{3}^{-1}-\mu_{3} \frac{b}{a} y_{2} y_{3}^{-1}=-\frac{b}{a} y_{1}-\frac{c}{b} y_{3}+y_{3}^{-1}$. Now apply (R2) to the first sum,

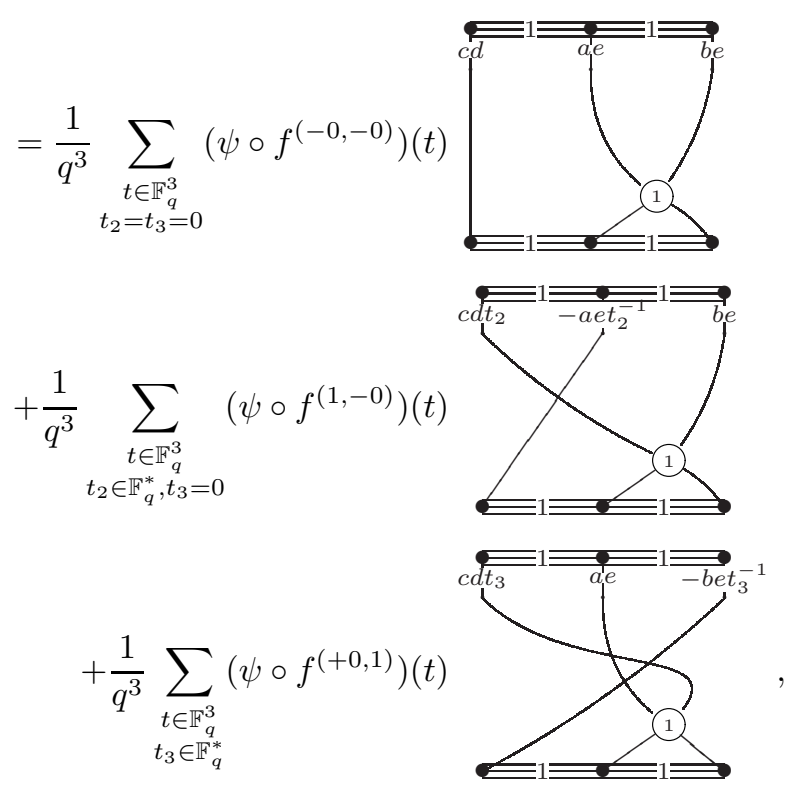

where $f^{(-0,-0)}=-\frac{b}{a} y_{1}-\frac{c}{b} y_{3}$ and $f^{(1,-0)}=\varphi\left(f^{(-0)}\right)+\mu_{1} \frac{a e}{c d} y_{2}^{-1}=-\frac{b}{a} y_{1}-y_{2}^{-1} y_{1}+$ $\frac{a e}{c d} y_{2}^{-1}$.

6. Theorem 5.1(4): Set $r=1$ with $w:=u_{2} s_{1}=1$ in the first sum, $w:=s_{1}$ in the second sum, and $w:=s_{1} s_{2} s_{1}$ in the third sum.

7. Theorem 5.1 (3a), (3a), (3b): In the first sum $\ell\left(s_{2} 1\right)>\ell(1)$, so apply (R1); in the second sum $\ell\left(s_{2} s_{1}\right)>\ell\left(s_{1}\right)$, so apply (ㅈ1); in the third sum, $\ell\left(s_{2} s_{1} s_{2} s_{1}\right)<$ 
$\ell\left(s_{1} s_{2} s_{1}\right)$, so paint $u_{1}\left(t_{1}\right)$ to get $u_{1}^{(1)}$,

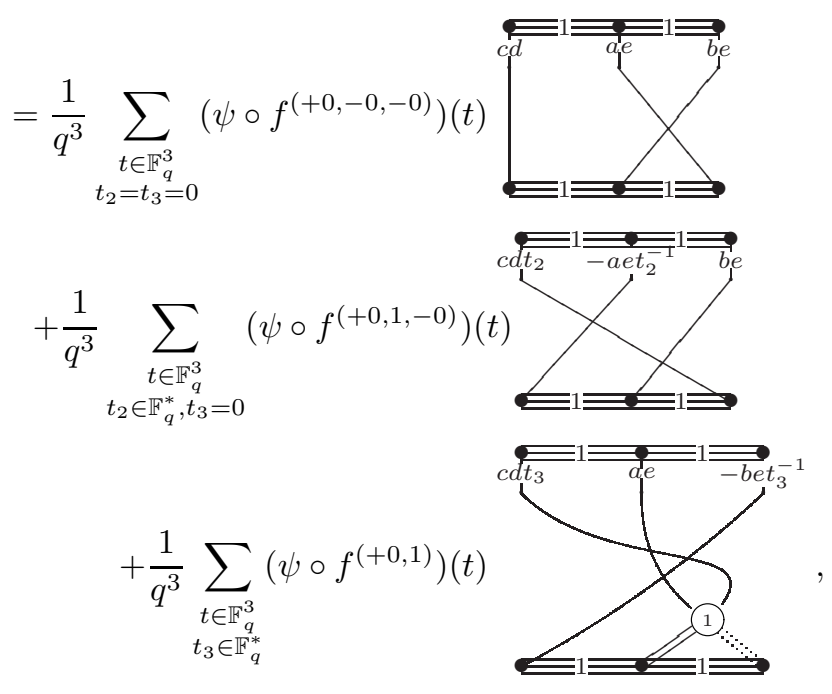

where $f^{(+0,-0,-0)}=-\frac{b}{a} y_{1}-\frac{c}{b} y_{3}+\frac{b}{a} y_{1}=-\frac{c}{b} y_{3}$ and $f^{(+0,1,-0)}=-\frac{b}{a} y_{1}+\frac{a e}{c d} y_{2}^{-1}-$ $y_{2}^{-1} y_{1}$. Now apply (R2) to the third sum,

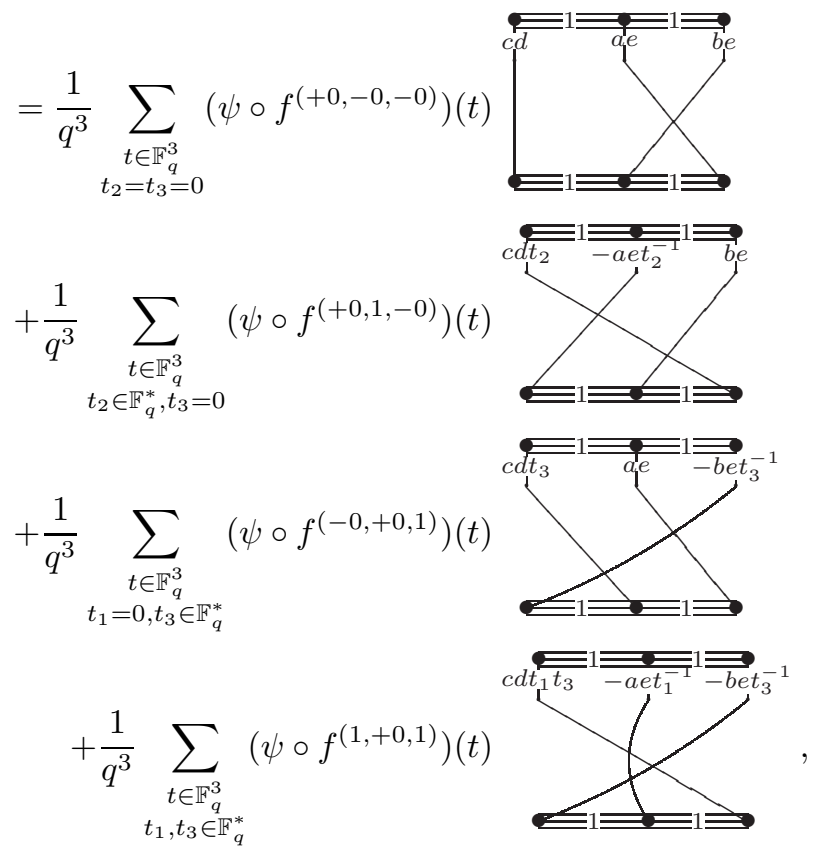

where $f^{(-0,+0,1)}=-\frac{c}{b} y_{3}+y_{3}^{-1}$ and

$$
f^{(1,+0,1)}=\varphi_{1}\left(f^{(+0,1)}\right)+\mu_{1} \frac{a e}{c d} y_{1}^{-1} y_{3}^{-1}=-\frac{b}{a} y_{1}-\frac{c}{b} y_{3}+y_{3}^{-1}+y_{1}^{-1}+\frac{a e}{c d} y_{1}^{-1} y_{3}^{-1} .
$$

8. Theorem 5.1(5): The first sum contains no elements of $N_{\mu}$, so set it to zero. The second sum contains elements of $N_{\mu}$ when $b e=-a e t_{2}^{-1}$, so set $t_{2}=-\frac{a}{b}$. The third sum contains elements of $N_{\mu}$ when $c d t_{3}=a e$, so set $t_{3}=\frac{a e}{c d}$. All the 
terms in the fourth sum are in $N_{\mu}$.

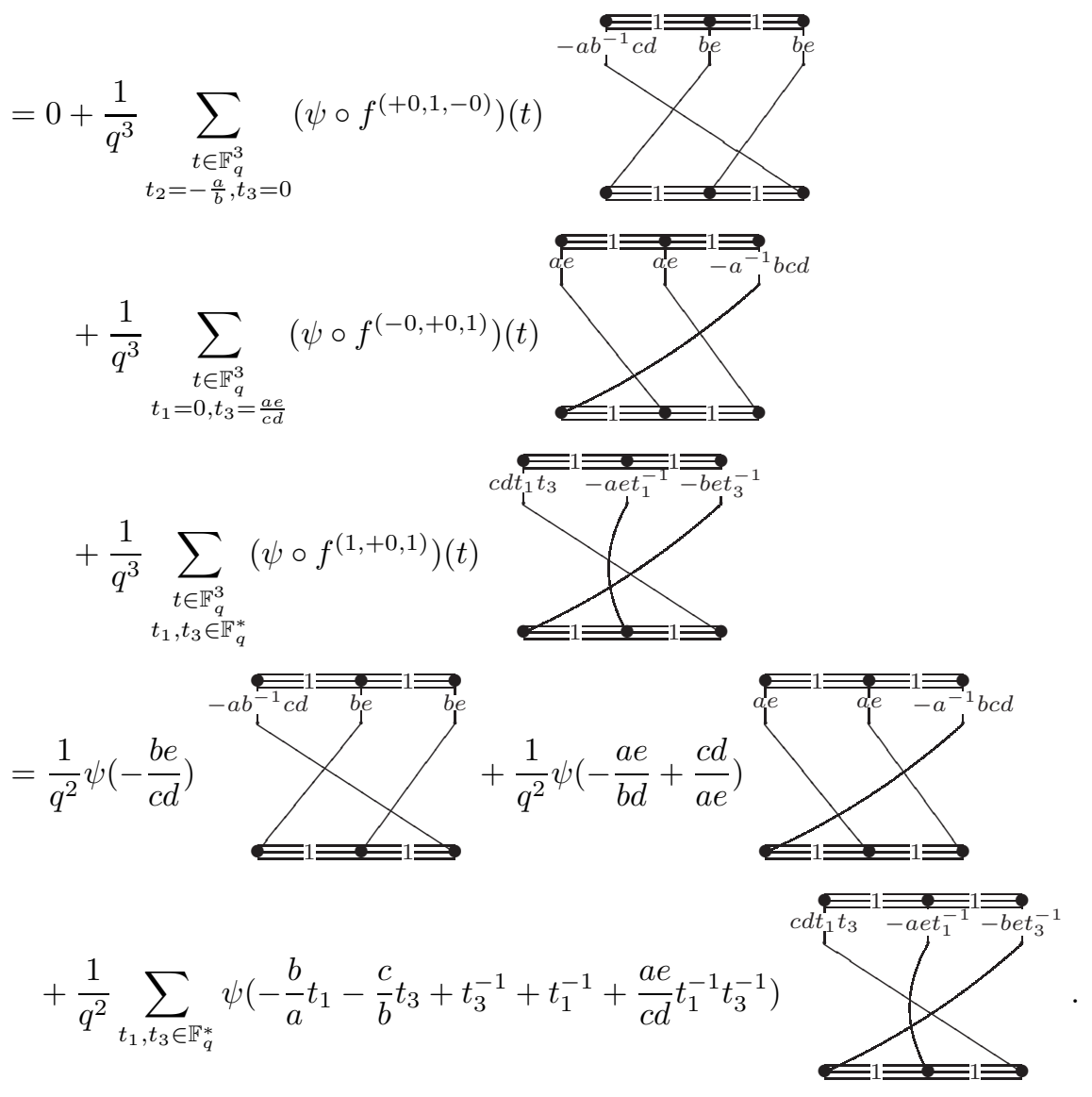

\section{ACKNOWLEDGMENTS}

Along with [Th, this paper is part of the author's Ph.D. thesis. In developing these results, the author has enjoyed the supportive environment of the University of Wisconsin-Madison mathematics department, the time supplied by several grants (VIGRE DMS-9819788, NSF DMS-0097977, and NSA MDA904-01-1-0032), and above all the patient help and insights of his advisor Arun Ram. The thoughtful comments and insights of an anonymous referee are also appreciated.

\section{REFERENCES}

[An] Andre, C. "Basic characters of the unitriangular group." Journal of Algebra 175 (1995): 287-319. MR1338979 (96h:20081a)

[ACDS] Arias-Castro, E.; Diaconis, P.; Stanley, R. "A super-class walk on upper-triangular matrices." June 2003 preprint.

[Bo] Bourbaki, N. Lie groups and Lie algebras. Chapters 4-6, New York: Springer Verlag, 2002. MR.1890629 (2003a:17001)

[Ch] Chang, B. "Decomposition of Gelfand-Graev characters of $G L_{n}(q)$." Communications in Algebra 4 (1976): 375-401. MR0401940 (53:5766)

$[\mathrm{Cu}] \quad$ Curtis, C. "A further refinement of the Bruhat decomposition." Proceedings of the American Mathematical Society 102 (1988): 37-42. MR0915711 (89d:20038) 
[CR] Curtis, C.; Reiner, I. Methods of Representation Theory, Vol. 1. New York: John Wiley and Sons, 1981. MR0632548 (82i:20001)

[CS] Curtis, C.; Shinoda, K. "Unitary Kloosterman sums and the Gelfand-Graev representation of $G L_{2} . "$ Journal of Algebra 216 (1999): 431-447. MR1693005 (2000h:11086)

[De] Demazure, M. "Schémas en groupes réductifs." Bulletin de la S.M.F. 93 (1965): 369-413. MR0197467 (33:5632)

[DM] Digne, F.; Michel, J. Representations of finite groups of Lie type. London Mathematical Society Student Texts 21, Cambridge University Press, 1991. MR1118841 (92g:20063)

[Hu] Humphreys, J.E. Introduction to Lie algebras and representation theory. New York: Springer Verlag, 1972. MR0323842 (48:2197)

[Ka1] Kawanaka, N. "Generalized Gelfand-Graev representations and Ennola duality." Advanced Studies in Pure Mathematics 6 (1985): 175-206. MR0803335 (87e:20075)

[Ka2] Kawanaka, N. "Generalized Gelfand-Graev representations of exceptional simple algebraic groups over a finite field I." Inventiones Mathematicae 84 (1986): 575-616. MR 0837529 (88a:20058)

[Ka3] Kawanaka, N. "Shintani lifting and Gelfand-Graev representations." Proceedings of Symposia in Pure Mathematics 47 (1987): 147-163. MR0933357 (89h:22037)

[Ra] Rainbolt, J. "The irreducible representations of the Hecke algebras constructed from the Gelfand-Graev representations of $G L(3, q)$ and $U(3, q)$." Communications in Algebra 30 (2002): 4085-4103. MR 1936457 (2003i:20007)

[St] Steinberg, R. Lectures on Chevalley groups. mimeographed notes, Yale University, 1967.

[Th] Thiem, N. "Unipotent Hecke algebras of $G L_{n}\left(\mathbb{F}_{q}\right)$." June 2003 preprint.

[Yo1] Yokonuma, T. "Sur le commutant d'une représentation d'un groupe de Chevalley fini." Journal of the Faculty of Science, University of Tokyo 15 (1968): 115-129. MR0248249 $(40: 1501)$

[Yo1.5] Yokonuma, T. "Complément au mémoire 'Sur le commutant d'une représentation d'un groupe de Chevalley fini'." Journal of the Faculty of Science, University of Tokyo 16 (1969): 147-148. MR0263947(41:8546)

[Yo2] Yokonuma, T. "Sur le commutant d'une représentation d'un groupe de Chevalley fini II." Journal of the Faculty of Science, University of Tokyo 16 (1969): 65-81. MR0263948 $(41: 8547)$

Department of Mathematics, Stanford University, Stanford, California 94305-2125 